\title{
HEXIM1 controls satellite cell expansion after injury to regulate skeletal muscle regeneration
}

Peng Hong, ${ }^{1,2}$ Kang Chen, ${ }^{3,4}$ Bihui Huang, ${ }^{5}$ Min Liu, ${ }^{6}$ Miao Cui, ${ }^{7}$ Inna Rozenberg, ${ }^{1}$ Brahim Chaqour, ${ }^{1,2}$ Xiaoyue Pan, ${ }^{1,2}$ Elisabeth R. Barton, ${ }^{8}$ Xian-Cheng Jiang, ${ }^{1,2}$ and M.A.Q. Siddiqui'1,2

\begin{abstract}
1Department of Cell Biology and ${ }^{2}$ Center for Cardiovascular and Muscle Research, State University of New York (SUNY) Downstate Medical Center, New York, New York, USA. ${ }^{3}$ Perinatology Research Branch, National Institute of Child Health and Human Development, NIH, Bethesda, Maryland, USA. ${ }^{4}$ Department of Obstetrics and Gynecology, Wayne State University School of Medicine, Detroit, Michigan, USA.

5Department of Cell and Developmental Biology, Weill Cornell Medical College, New York, New York, USA. ${ }^{6}$ Department of Physiology, Perelman School of Medicine, University of Pennsylvania, Philadelphia, Pennsylvania, USA. ${ }^{7}$ Division of Clinical Immunology, Department of Medicine, Mount Sinai Medical Center, New York, New York, USA. ${ }^{8}$ Department of Anatomy and Cell Biology, University of Pennsylvania School of Dental Medicine, Philadelphia, Pennsylvania, USA.
\end{abstract}

\begin{abstract}
The native capacity of adult skeletal muscles to regenerate is vital to the recovery from physical injuries and dystrophic diseases. Currently, the development of therapeutic interventions has been hindered by the complex regulatory network underlying the process of muscle regeneration. Using a mouse model of skeletal muscle regeneration after injury, we identified hexamethylene bisacetamide inducible 1 (HEXIM1, also referred to as CLP-1), the inhibitory component of the positive transcription elongation factor $b(P-T E F b)$ complex, as a pivotal regulator of skeletal muscle regeneration. Hexim1-haplodeficient muscles exhibited greater mass and preserved function compared with those of WT muscles after injury, as a result of enhanced expansion of satellite cells. Transplanted Hexim1-haplodeficient satellite cells expanded and improved muscle regeneration more effectively than WT satellite cells. Conversely, HEXIM1 overexpression restrained satellite cell proliferation and impeded muscle regeneration. Mechanistically, dissociation of HEXIM1 from P-TEFb and subsequent activation of $\mathrm{P}-\mathrm{TEFb}$ are required for satellite cell proliferation and the prevention of early myogenic differentiation. These findings suggest a crucial role for the HEXIM1/P-TEFb pathway in the regulation of satellite cell-mediated muscle regeneration and identify HEXIM1 as a potential therapeutic target for degenerative muscular diseases.
\end{abstract}

\section{Introduction}

Skeletal muscle possesses remarkable regenerative capacity for the repair of muscle damage in injuries or muscular disorders $(1,2)$. Loss of muscle regenerative capacity, such as that seen in dystrophic muscle diseases characterized by progressive muscle degeneration and weakness, leads to reduced mobility and premature death (3). Fibrotic muscle regeneration after ineffective treatment of sports injuries also causes deleterious consequences, such as chronic pain and permanent loss of muscle strength (4). Unlike Duchenne muscular dystrophy, caused by a single-gene mutation of $D M D$ that can be effectively rescued by genetic approaches (5, 6), diverse pathways are involved in the loss of muscle regenerative capacity, which complicates therapeutic interventions (7-9).

In addition to exploiting regulatory pathways to promote muscle regeneration, recent studies have also focused on transplanting progenitor cells, including satellite cells, mesoangioblasts, and embryonic stem cells (10-12). Among them, satellite cells, the skeletal muscle progenitors, are the major contributor to skeletal muscle regeneration (13). Experimental studies in mice show that depletion of satellite cells by genetic modification impedes muscle regeneration (14), while transplanting exogenous satellite cells into injured or dystrophic muscles improves the outcome of regeneration $(15,16)$. Most satellite cells are quiescent in healthy adult skeletal muscles but can undergo rapid expansion upon muscle injury to serve as a robust myogenic source for the regeneration of myofibers $(1,2)$. This injury response is initiated by various

Conflict of interest: The authors have declared that no conflict of interest exists. Citation for this article: J Clin Invest. 2012;122(11):3873-3887. doi:10.1172/JCI62818. cytokines secreted by infiltrating immune cells and mediated by a network of myogenic transcription factors, including Pax $3 / 7$, Myf5, MyoD, and MASTR (17-19). Although satellite cells are the native progenitors for muscle regeneration, the efficacy of satellite cell therapy has been limited due to poor survival, self-renewal, and migration of transplanted satellite cells $(10,20,21)$. Recently, a number of other cell types, such as Sca- $1^{+}$stem cells, pericytes, and side population cells, have been shown to possess myogenic potential in muscle development or regeneration and, after further evaluation of their efficacy, might serve as an alternative to satellite cells in therapeutic approaches (22-25).

Hexamethylene bisacetamide inducible 1 (HEXIM1, also known as CLP-1) has been characterized as an inhibitory component of the positive transcription elongation factor $\mathrm{b}$ (P-TEFb) complex $(26,27)$. Cyclin-dependent kinase 9 (CDK9), the kinase component of P-TEFb, is activated by dissociation of HEXIM1 from the cofactor cyclin T (27). After transcription initiation, RNA polymerase II (Pol II) often pauses at a promoter-proximal region, and the pausing escape of $\mathrm{Pol}$ II and subsequent productive elongation requires CDK9 to phosphorylate Ser2 within the carboxylterminal domain (CTD) of the largest subunit of Pol II (28). The recruitment of P-TEFb to Pol II could be mediated by a general transcription factor, Brd4, or certain sequence-specific transcription factors, including Myc and NF-kB (29-31). Therefore, manipulation of P-TEFb activity could give both global and gene-specific effects, yet whole-genome profiling following such manipulation in a specific cell type has not been reported. P-TEFb has been reported to regulate the function of key myogenic transcription factors MyoD and MEF2 (32-34), while P-TEFb activity is globally 

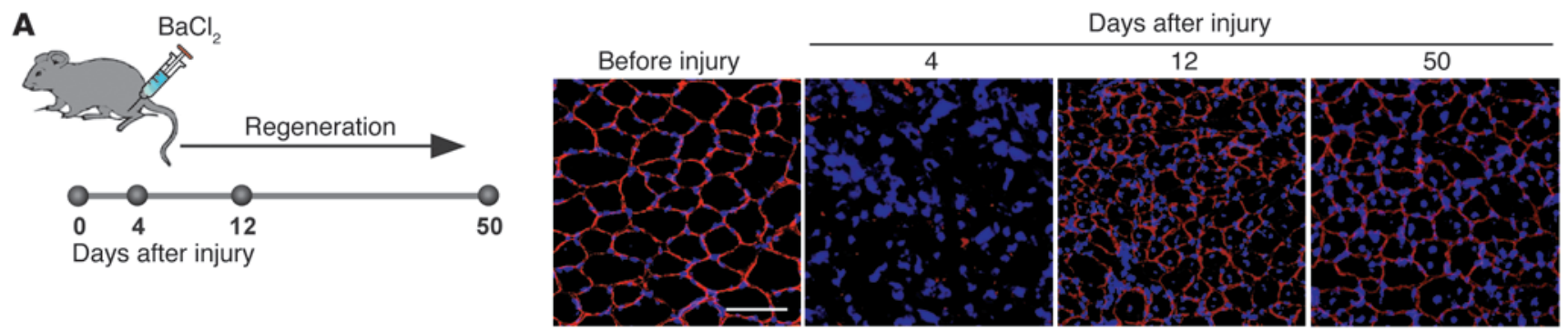

Dystrophin/DAPI

B

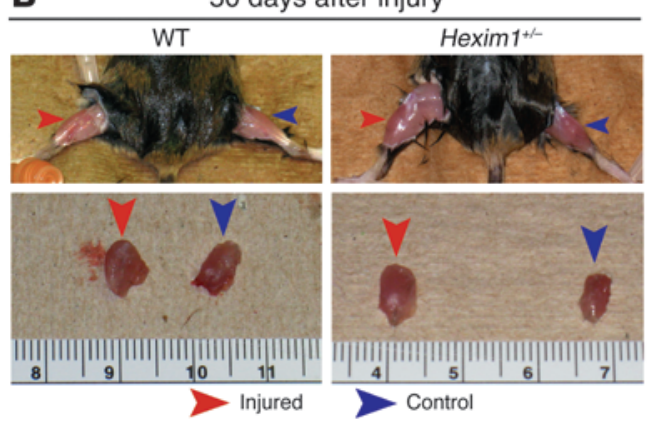

C

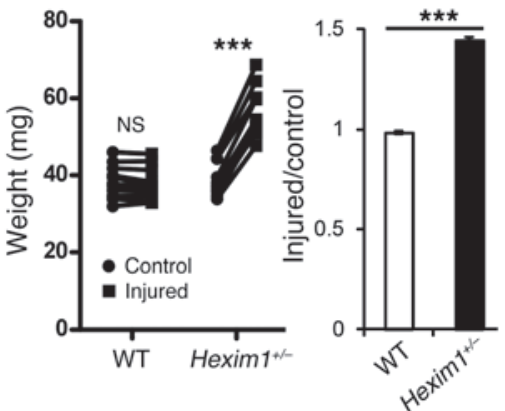

D

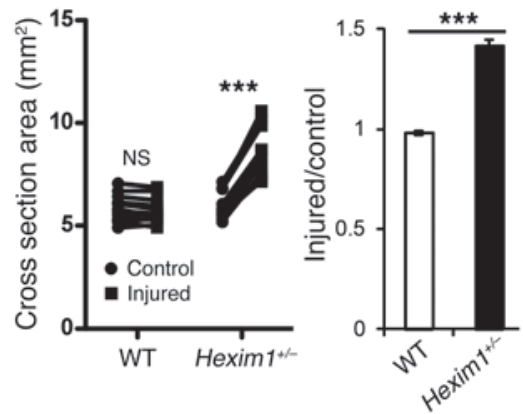

G

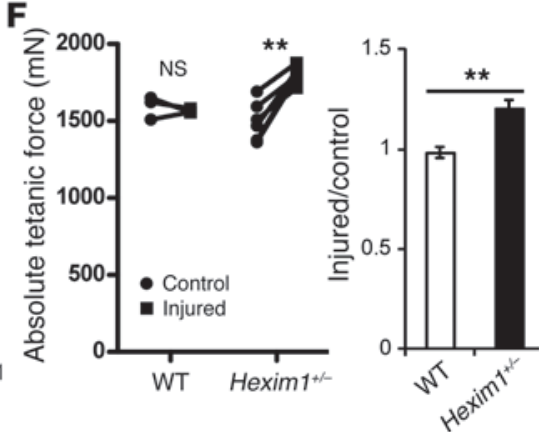

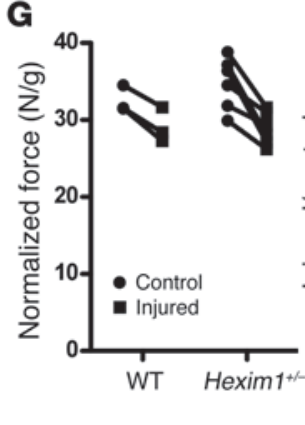

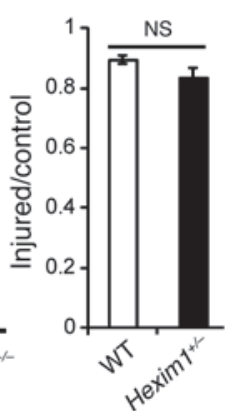

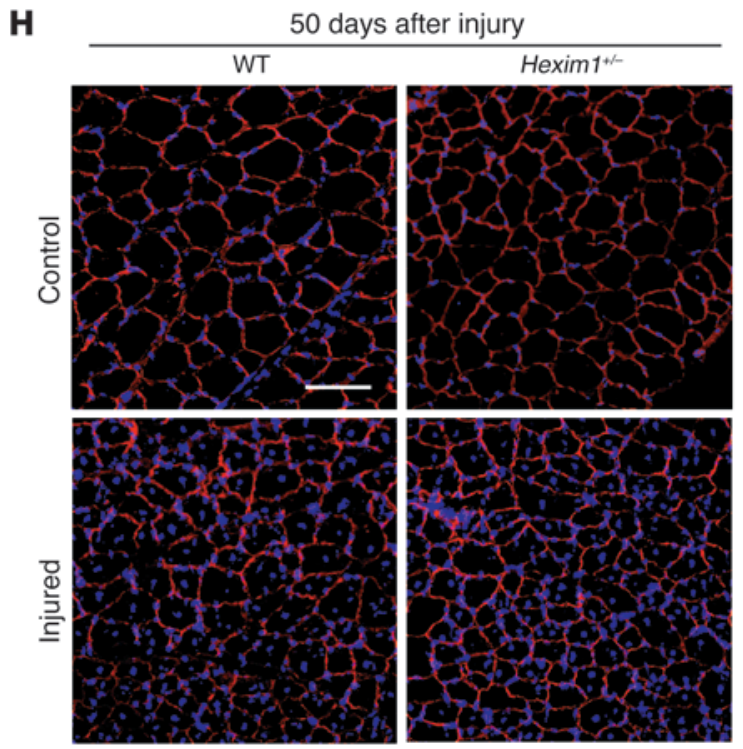

Dystrophin/DAPI

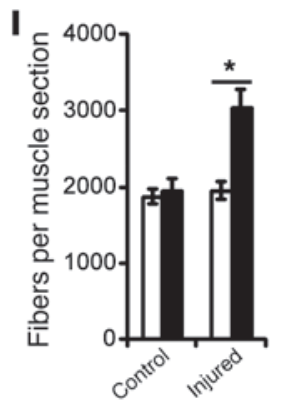

K

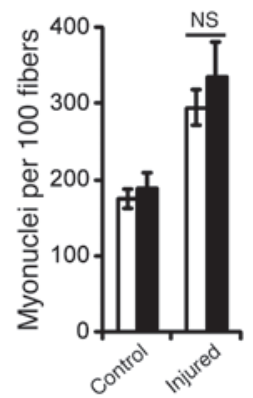

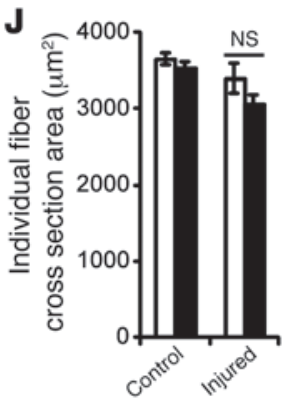

$\square W T$

-Hexim $1^{+/-}$
$\mathbf{L}$

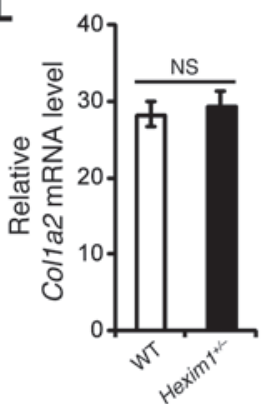




\section{Figure 1}

Hexim $1^{+/}$muscles exhibit larger size and enhanced function after regeneration. (A) Model of $\mathrm{BaCl}_{2}$-induced muscle injury. Dystrophin staining outlines muscle sarcomeres. Scale bar: $80 \mu \mathrm{m}$. (B) Injured and contralateral control TA muscles 50 days after injury. (C) Muscle wet weights, (D) cross-section areas, and (C and D) their injured-tocontrol ratios 50 days after injury $(n=12)$. (E) Representative plots of tetanic force production of injured and contralateral control TA muscles. (F) Absolute tetanic forces, (G) normalized tetanic forces, and (F and G) their injured-to-control ratios 10 weeks after injury ( $n=3$ [WT] or 6 $\left[\right.$ Hexim $\left.\left.1^{+-}\right]\right)$. (H) Muscle sections 50 days after injury stained for dystrophin (red). Scale bar: $80 \mu \mathrm{m}$. (I) Average numbers of muscle fibers per section, $(\mathbf{J})$ average individual myofiber sizes, and $(\mathbf{K})$ average numbers of myonuclei per 100 muscle fibers 50 days after injury $(n=6)$. (L) Col1a2 mRNA levels 4 days after injury $(n=3)$. Statistical significance in scatter plots was assessed by a 2-tailed paired Student's $t$ test. ${ }^{\star} P<0.05 ;{ }^{* \star} P<0.01 ;{ }^{* \star} P<0.001$.

suppressed in most adult stem cells, including satellite cells, due to their low demand of mRNA synthesis (35). We have previously shown that haplodeficiency of Hexim1 led to inadequate P-TEFb inhibition under serum starvation and impaired myogenic differentiation of C2C12 cells in vitro (36).

In this study, we used an established model of skeletal muscle regeneration after injury $(37,38)$ to demonstrate that modulation of HEXIM1, either in vivo or in transplanted satellite cells, enhanced skeletal muscle regeneration after injury. We also show that the expansion of the satellite cell pool, which provides the myogenic source for muscle regeneration, is directly controlled by the dynamic inhibition of P-TEFb mediated by HEXIM1.

\section{Results}

Hexim $1^{+/}$skeletal muscles exhibit enhanced regeneration after injury. Since Hexim 1 haplodeficiency in $\mathrm{C} 2 \mathrm{C} 12$ cells prevents myogenic differentiation, which is one of the central events in skeletal muscle regeneration, we hypothesized that HEXIM1 may regulate skeletal muscle regeneration after injury by modulating satellite cell function. To test this hypothesis, we used a model of tibialis anterior (TA) muscle regeneration after $\mathrm{BaCl}_{2}$-induced muscle injury in mice (Figure 1A). We first validated that HEXIM1 was present in the nuclei of satellite cells (Supplemental Figure 1A; supplemental material available online with this article; doi:10.1172/ JCI62818DS1) and that the cellular HEXIM1 protein level was reduced by approximately half in Hexim $1^{+/-}$mice (Supplemental Figure 1, B and C). After $\mathrm{BaCl}_{2}$-induced injury, complete and homogeneous destruction of TA muscle sarcomere structures was seen 4 days after injury, while nascent myofibers were regenerated 12 days after injury (Figure 1A and Supplemental Figure 1D). This regenerative process typically reached homeostasis 50 days after injury (ref. 38, Figure 1A, and Supplemental Figure 1D).

Skeletal muscles in Hexim $1^{+/-}$mice developed with normal structures and grew at a rate comparable to that of WT muscles (Supplemental Figure 1, D and E). However, unlike WT mice that regenerate injured muscles to a comparable size and structure of that of their contralateral control muscles, Hexim $1^{+/-}$mice were capable of regenerating bigger muscles with higher weights and larger crosssection areas than those of their contralateral muscles after injury (Figure 1, B-D). Injured-to-control ratios of wet weights were $49 \%$ higher in Hexim $1^{+/-}$muscles $(1.44 \pm 0.018)$ than in WT controls $(0.99 \pm 0.0068) 50$ days after injury $\left(P=1.25 \times 10^{-12}\right)$ (Figure $\left.1 \mathrm{C}\right)$.
Injured-to-control ratios of cross-section areas were $44 \%$ larger in Hexim $1^{+/-}$mice $(1.42 \pm 0.026)$ than in WT controls $(0.98 \pm 0.0075)$ 50 days after injury $\left(P=6.71 \times 10^{-10}\right)$ (Figure $\left.1 \mathrm{D}\right)$.

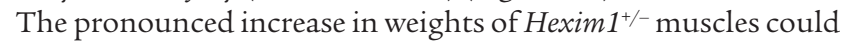
arise from compensatory hypertrophy due to loss of force-generating capacity. To address this possibility, isometric tetanic forces were measured in injured and contralateral control TA muscles of both WT and Hexim $1^{+/-}$mice 10 weeks after injury. Absolute forces increased in injured Hexim $1^{+/-}$muscles $(1,799 \pm 22 \mathrm{mN})$ compared with those in contralateral control muscles $(1,500 \pm 52$ $\mathrm{mN})(P=0.0014)$, which differed from the lack of increased force production in injured WT muscles $(1,565 \pm 8.4 \mathrm{mN}$ versus $1,593 \pm 43$ $\mathrm{mN}, P=0.56$ ) (Figure 1, E and F). Injured-to-control ratios of tetanic forces were correspondingly higher in Hexim $1^{+/-}$mice $(1.21 \pm 0.038$ versus $0.98 \pm 0.026, P=0.002)$ (Figure $1 F)$. The increase in forces of injured Hexim $1^{+/-}$muscles was proportional to the increase in weights, such that the normalized tetanic force, defined as the absolute tetanic force divided by the muscle weight, showed comparable injured-to-control ratios between Hexim $1^{+/-}$and WT muscles $(0.84 \pm 0.031$ versus $0.89 \pm 0.015$, $P=0.13$ ) (Figure $1 \mathrm{G})$. Hence, the increase in muscle weights following regeneration in $\mathrm{Hexim}^{+/-}$mice contributed to the potential force production of the muscles, rather than producing nonfunctional hypertrophy, and so the enhanced regenerative capacity also leads to enhanced strength.

Sarcolemma-specific dystrophin staining of transverse muscle sections (Figure $1 \mathrm{H})$ revealed $56 \%$ more myofibers $(P=0.019)$ in injured Hexim $1^{+/-}$muscles than in injured WT controls 50 days after injury (Figure 1I). No difference in average myofiber sizes was observed between the 2 genotypes $(P=0.19)$ (Figure 1J), indicating a lack of hypertrophy. The numbers of myonuclei per myofiber were comparable between the 2 genotypes (Figure $1 \mathrm{~K}$ ). There was no elevation of collagen I $\alpha 2$ (Col1a2) mRNA 4 days after injury (Figure 1L) and no increase of extracellular space in injured Hexim $1^{+/-}$muscles 12 and 50 days after injury (Figure $1 \mathrm{H}$ and Supplemental Figure 1D). This indicates that fibrosis in Hexim $1^{+/-}$ muscles is not enhanced during regeneration and does not contribute to the increased mass and size of regenerated muscles. Vegfa mRNA and VEGF protein levels in injured muscles 4 days after injury were comparable between the 2 genotypes (Supplemental Figure 2, A and B), and vessel counting showed no difference in capillary density 50 days after injury (Supplemental Figure 2, C and D), indicating normal angiogenesis in Hexim $1^{+/-}$muscles. These data demonstrate that skeletal muscles of Hexim $1^{+/-}$mice possess enhanced regenerative capacity, as achieved by the regeneration of more myofibers after injury.

Regenerating Hexim $1^{+-}$muscles harbor more satellite cells. As satellite cells are the main source of skeletal muscle regeneration, we quantitated them on muscle sections based on their specific marker, Pax7 (ref. 13 and Figure 2A). Satellite cell number normalized to myofiber length was normal in intact and fully regenerated Hexim $1^{+/-}$ muscles (Supplemental Figure 3A), indicating normal maintenance of satellite cells. In contrast, injured $\mathrm{Hexim} 1^{+/-}$muscles harbored 2 -fold more satellite cells per section ( $492 \pm 28$ cells per muscle section) than injured WT controls ( $247 \pm 15$ cells per muscle section) 4 days after injury $\left(P=7.85 \times 10^{-6}\right)$ and continued to contain more satellite cells 12 and 50 days after injury (Figure 2B). The density of satellite cells in injured Hexim $1^{+/-}$muscles was higher 4 and 12 days after injury and returned to the WT level 50 days after injury (Figure 2C). Homeostatic expansion of satellite cells in Hexim $1^{+/-}$mus- 
A

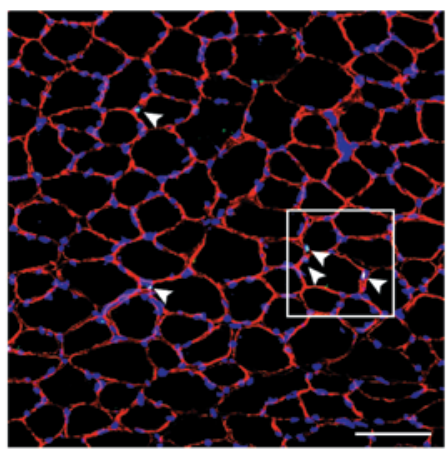

Pax7/Dystrophin/DAPI
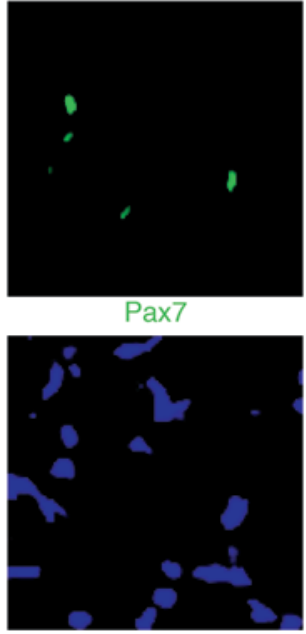

B

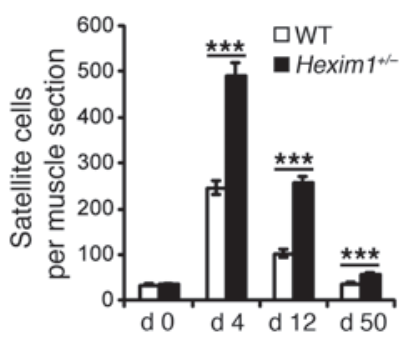

C

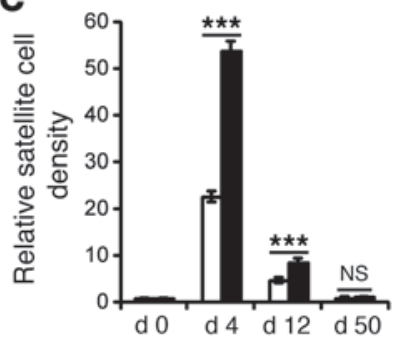

E

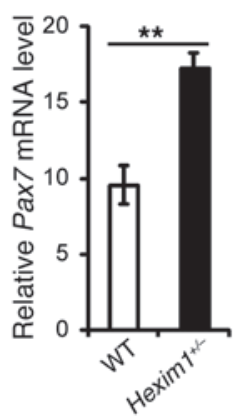

DAPI
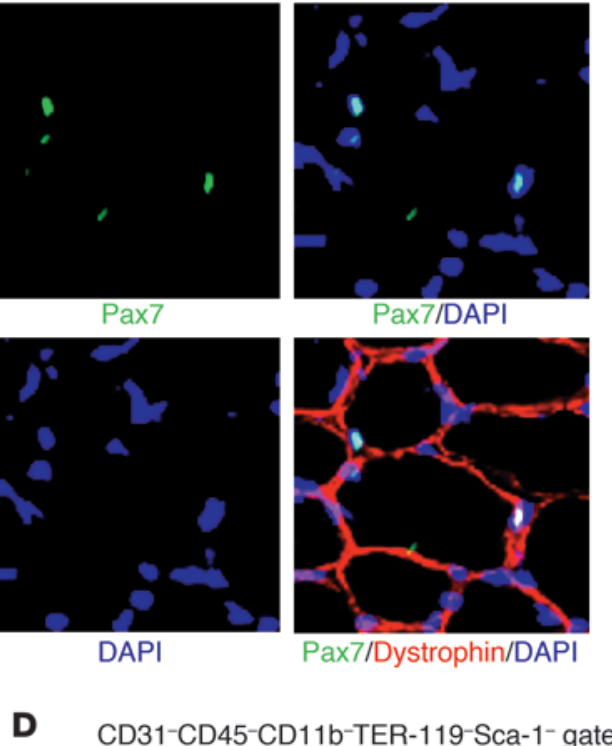

Pax7/DAPI

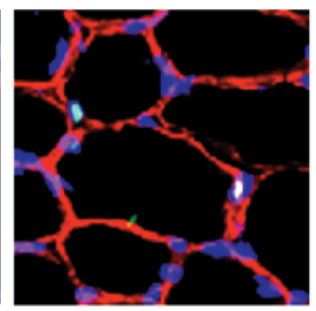

Pax7/Dystrophin/DAPI

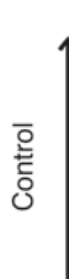

D31-CD45-CD11b-TER-119-Sca-1- gate
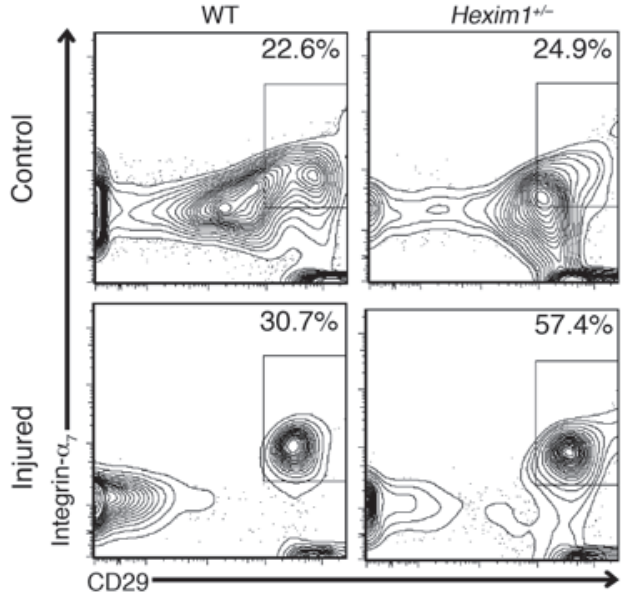

\section{Figure 2}

Hexim $1^{+/-}$muscles harbor more satellite cells during regeneration. (A) Sample section stained for Pax7 (green) and dystrophin (red). Arrowheads indicate myofiber-associated $\mathrm{Pax} 7^{+}$satellite cells. Colocalization of Pax7 and DAPI staining in the nuclei of satellite cells from the boxed region is shown to the right. Scale bar: $80 \mu \mathrm{m}$. (B) Average numbers of $\mathrm{Pax} 7^{+}$satellite cells per section and (C) relative satellite cell densities defined as the ratio of satellite cell number to the crosssection area and normalized to the ratio in WT muscles before injury. Results were calculated from the average values of 3 muscle sections per mouse, and 6 mice were analyzed. (D) Satellite cell percentages in injured and contralateral control muscles 4 days after injury. Plots were representative of 6 independent experiments. (E) Pax7 mRNA levels 4 days after injury $(n=3)$. (F) Ccnd 1 mRNA levels 4 days after injury $(n=3) .{ }^{* *} P<0.05$; ${ }^{\star * *} P<0.001$ cles was ruled not to be a contributing factor for the enlargement of the satellite cell pool (Supplemental Figure 3B).

Flow cytometry, based on CD29 (integrin $\beta_{1}$ ) and integrin $\alpha_{7}$ expression that defines total satellite cells $(2,39)$, showed an approximately 2 -fold higher satellite cell percentage in injured Hexim $1^{+/-}$muscles than in injured WT muscles 4 days after injury (Supplemental Figure 4A and Figure 2D). Survival was comparable between WT and Hexim $1^{+/-}$satellite cells 1 day after injury (Supplemental Figure 4B). Control muscles showed no difference in satellite cell percentage between the 2 genotypes (Figure 2D). Flow cytometric analyses of other satellite cell markers, including CD34, integrin $\alpha_{7}$, CD29 and CXCR4 $(40,41)$, showed similar satellite cell enrichment in injured Hexim $1^{+/-}$muscles 4 days after injury (Supplemental Figure 4, C and D). Moreover, mRNA of Pax7, the transcription factor exclusively expressed by satellite cells within muscles (13), was 2-fold higher in injured Hexim $1^{+/-}$muscles than in injured WT controls 4 days after injury (Figure 2E), which corroborates the above findings. Of note, infiltration of hematopoi- 
etic cells was comparable in WT and Hexim $1^{+/-}$muscles 4 days after injury (Supplemental Figure 4E).

Haplodeficiency of Hexim1 enhances satellite cell proliferation after injury. The elevated mRNA level of cyclin D1 (Ccnd1), 2.5-fold higher in $\mathrm{Hexim}^{+/-}$muscles than in WT muscles, implies that Hexim $^{+/-}$satellite cells underwent enhanced proliferation during regeneration (Figure $2 \mathrm{~F}$ ). To ascertain this possibility, we sorted WT and Hexim $1^{+/-}$satellite cells from injured TA muscles at 4 days after injury when the total satellite cell number peaked and analyzed their cell cycle status based on DNA and RNA contents. Flow cytometry revealed approximately $30 \%$ more $\mathrm{Hexim}^{1^{+/}}$satellite cells in $S / G_{2} / M$ phases, demonstrating enhanced proliferation of Hexim $1^{+/-}$satellite cells (Figure $3 \mathrm{~A}$ ). In contrast, Sca- $1^{+}$stem cells, which could also contribute to muscle regeneration (25), proliferated more slowly than satellite cells, with no difference in the percentage of cells in $S / G_{2} / M$ phases between the 2 genotypes (Figure 3B). Analysis of other cell populations, including pericytes, endothelial cells, and CD $45^{+}$hematopoietic cells, revealed similarly low proliferation rate $\left(<5 \% \mathrm{~S} / \mathrm{G}_{2} / \mathrm{M}\right.$ cells $)$ and comparable cell cycle composition between WT and Hexim $1^{+/-}$muscles 4 days after injury (data not shown). Apoptosis of satellite cells or Sca- $1^{+}$cells 4 days after injury was comparable between the 2 genotypes (Figure 3, C and D). Flow cytometric analysis, which showed higher percentages of satellite cells and comparable percentages of Sca- $1^{+}$cells in Hexim $1^{+-}$muscles 4 days after injury, confirmed the cell proliferation data (Figure 3, E and F). Of note, the percentages of side population cells, which are typically Sca- $1^{+}$, also displayed no difference between the 2 genotypes 4 days after injury (Supplemental Figure 4F). Together, these data demonstrate that haplodeficiency of Hexim 1 predominantly enhances satellite cell proliferation during early stages of muscle regeneration.

Transplanted Hexim $1^{+/-}$satellite cells undergo enhanced expansion and improve muscle regeneration. Since skeletal muscle regeneration requires the cooperative effort of various cell populations residing in or infiltrating into the regenerating muscle (1), it is important to verify whether haplodeficiency of Hexim 1 in satellite cells is sufficient to enhance muscle regeneration. We therefore sorted quiescent WT and Hexim $1^{+-}$satellite cells based on their expression of CD29, syndecan-4, and CXCR4 (CD184) (40, 42) (Supplemental Figure 5A) and examined their proliferative and regenerative capacity in vivo by transplanting them into WT injured TA muscles (Figure 4A). Sorted cells were verified to uniformly express the satellite cell-specific marker Pax7 and lack the expression of the myoblast marker MyoD (Figure $4 \mathrm{~B}$ ) and were predominantly in $\mathrm{G}_{0}$ phase (Supplemental Figure 5B). To track transplanted cells and measure their expansion in the host, sorted Hexim $1^{+/-}$and WT cells were equally labeled with PKH26 immediately before transplantation (Supplemental Figure 5C). After transplantation of an equal number of labeled Hexim $1^{+/-}$ or WT cells into injured WT muscles, transplanted cells proliferated in response to injury, and PKH26 signal decreased by half after each cell division, which permitted the analysis of cell cycle progression. Transplanted cells were distinguished from unlabeled host cells by PKH26 gating, based on contralateral untransplanted TA muscles (Supplemental Figure 5D). No significant difference in cell death was detected between transplanted WT and Hexim $1^{+/-}$cells 24 hours after transplantation (Supplemental Figure 5E). Hexim $1^{+/}$cells proliferated more extensively than WT cells, as evidenced by their lower PKH26 signals (Figure 4C) and higher percentages of phospho-histone $\mathrm{H}^{+}$cells (Figure 4D and Supplemental Figure 5F). Two-fold more labeled cells were recorded in muscles receiving Hexim $1^{+/-}$ satellite cells than in muscles receiving WT satellite cells, further demonstrating the enhanced proliferative capacity of Hexim $1^{+/-}$ satellite cells in vivo (Figure 4E). Furthermore, transplantation of Hexim $1^{+/-}$satellite cells enhanced the expansion of the host's satellite cell pool more effectively than transplantation of WT satellite cells (Figure 4F). After the completion of muscle regeneration at 50 days after injury, muscles that received Hexim $1^{+/-}$satellite cells had significantly larger sizes and higher injured-to-control ratios of wet weights $(1.52 \pm 0.055)$ than those of muscles that received WT satellite cells $(1.17 \pm 0.030)\left(P=5.88 \times 10^{-4}\right)$ (Figure $4, \mathrm{G}$ and $\left.\mathrm{H}\right)$; albeit, both had normal muscle structure and myonuclei deposition (Figure 4I). These data demonstrate that the superior proliferative capacity and regenerative potential of Hexim $1^{+/-}$satellite cells is functionally sufficient to enhance the expansion of the satellite cell pool and improve muscle regeneration.

Hexim $1^{+-}$satellite cells exhibit enhanced proliferation over differentiation. Similar to their enhanced proliferation and expansion after injury in vivo (Supplemental Figure 6A), Hexim 1 ${ }^{+/-}$satellite cell colonies expanded more rapidly than WT colonies in growth media (GM) (Figure 5A). Measurement of PKH26 signal indicated that Hexim $1^{+/-}$satellite cells underwent more rapid activation at day 1 and more proliferation at day 4 (Figure 5B). At day 3 , these cells generated heterogeneous progeny with differential expressions of Pax7 and MyoD (Figure 5C), reflecting a gradual loss of satellite cell identity with simultaneous differentiation during expansion (43). Two-fold more cells remained $\mathrm{Pax}^{+} \mathrm{MyoD}^{-}$in $\mathrm{Hexim} 1^{+/-}$cultures than in WT cultures, and fewer Pax $7^{-} \mathrm{MyoD}^{+}$cells were found in Hexim 1 $^{+/}$- cultures at day 3 (Figure 5D). Myod1 mRNA was not lower in $\mathrm{Hexim}^{+/-}$muscles than in WT controls during regeneration (Supplemental Figure 6B), ruling out the possibility of the loss of function of MyoD in Hexim $1^{+-}$satellite cells.

Consistent with their enhanced proliferative capacity, differentiation of Hexim $1^{+/}$satellite cells was delayed upon injury. Hexim $1^{+-}$ muscles contained fewer differentiated (myogenin ${ }^{+}$) myoblasts (Figure 5E), lower desmin and myosin heavy chain (MHC) protein levels (Figure 5F), and 75\% less Myb3 (embryonic myosin heavy chain [eMHC]) mRNA (Figure 5G) than WT controls 4 days after injury. More than $90 \%$ of transplanted Hexim $1^{+/-}$satellite cells retained the expression of satellite cell markers after 3 days of expansion after injury, while approximately half of transplanted WT cells downregulated satellite cell markers (Figure 5H). Myogenin staining of $\mathrm{PKH} 26^{+}$cells confirmed less differentiation of transplanted Hexim 1 ${ }^{+/}$satellite cells 3 days after injury (Figure 5I). The structure and size of regenerated myofibers became comparable between the 2 genotypes 12 days after injury when differentiation of satellite cells and myofiber growth became dominant (Supplemental Figure 6C). This indicates that myogenic differentiation capacity of Hexim $1^{+/-}$satellite cells is not compromised.

Hexamethylene bisacetamide-induced HEXIM1 expression restrains satellite cell proliferation. To examine the consequence of HEXIM1 induction on satellite cell proliferation, we added hexamethylene bisacetamide (HMBA), the specific inducer of HEXIM1 expression $(27,44)$, in satellite cell cultures (Figure 6A). Upon treatment with HMBA in GM, proliferation was attenuated (Figure 6B) while differentiation was significantly accelerated (Figure 6, C and D). When switched into low-mitogen differentiation media (DM), Hexim $1^{+/-}$satellite cell cultures differentiated more slowly than WT cultures and were restored to normal differentiation rate by HMBA treatment, as measured by the expression of the myogenic differentiation marker myogenin (Figure 6E). No enhanced apop- 
A
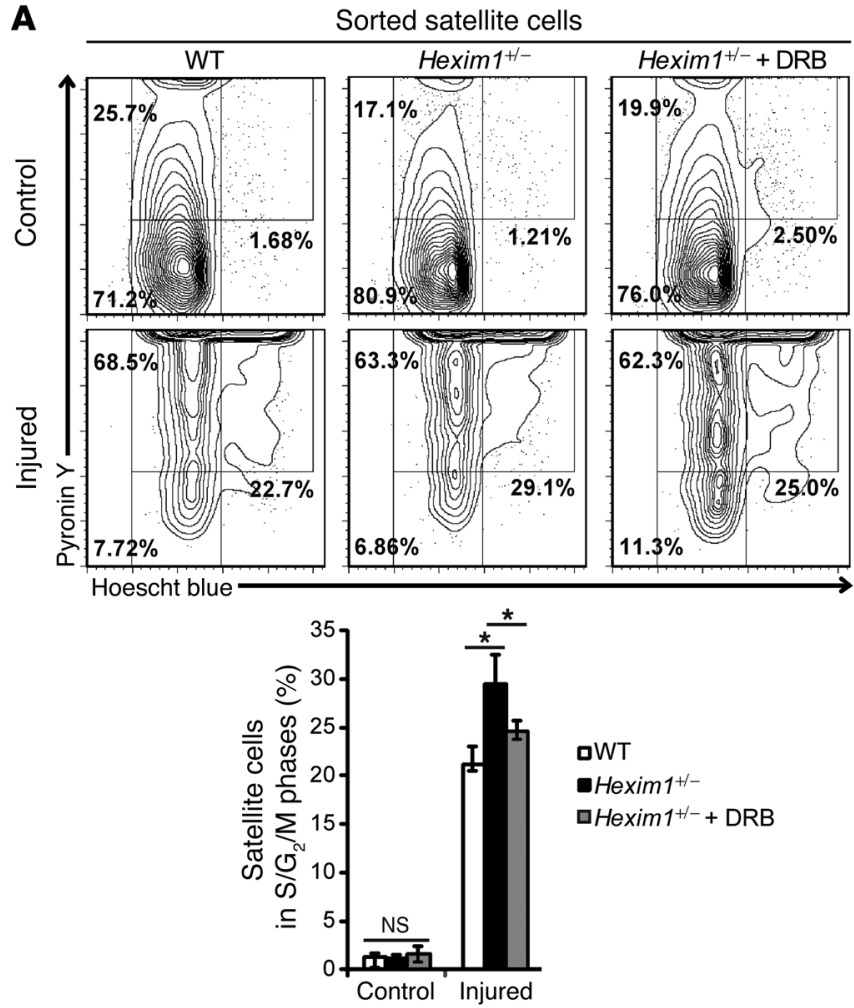

C

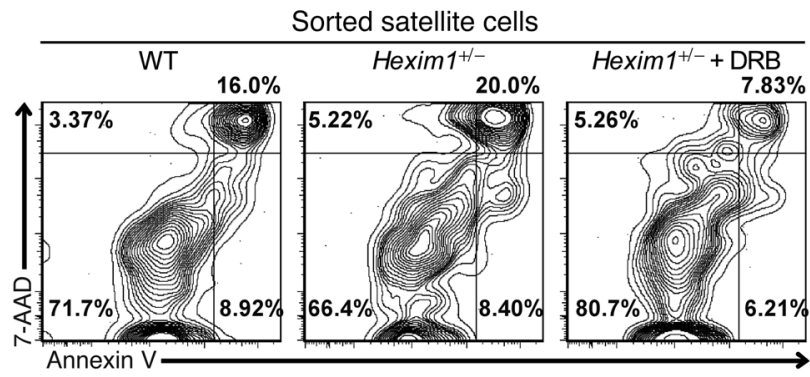

E

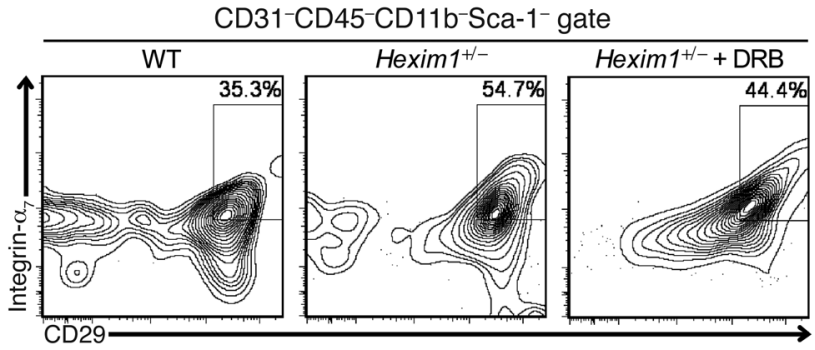

B
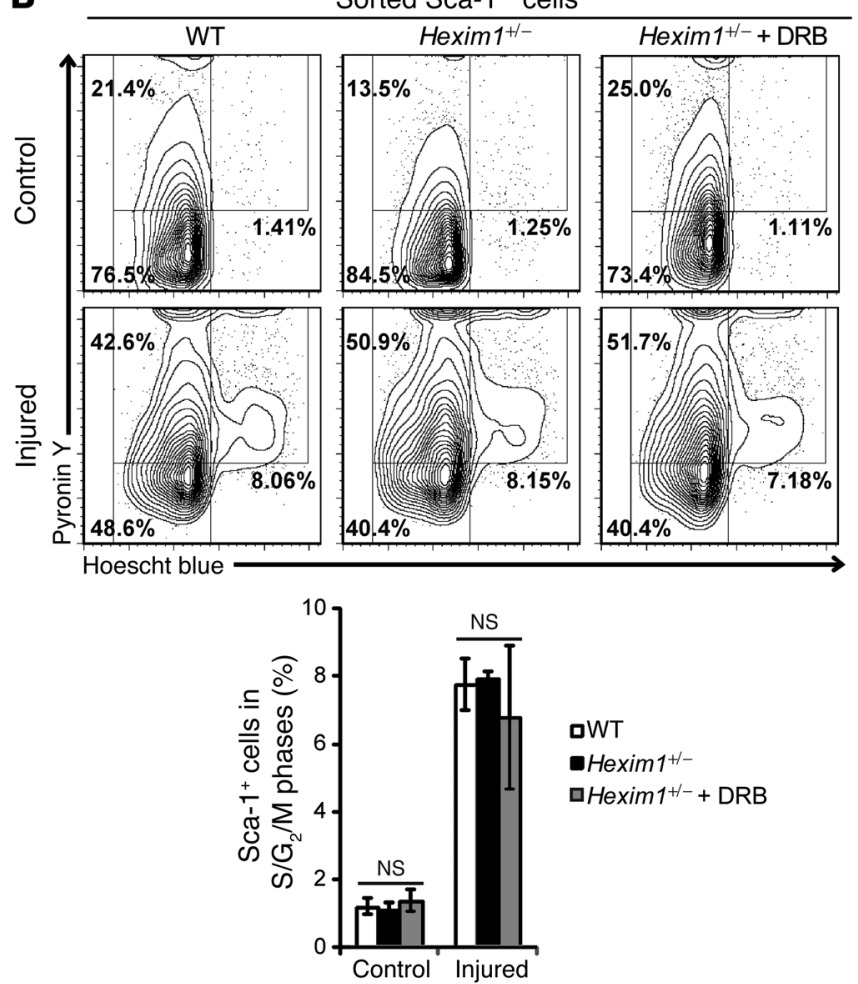

D

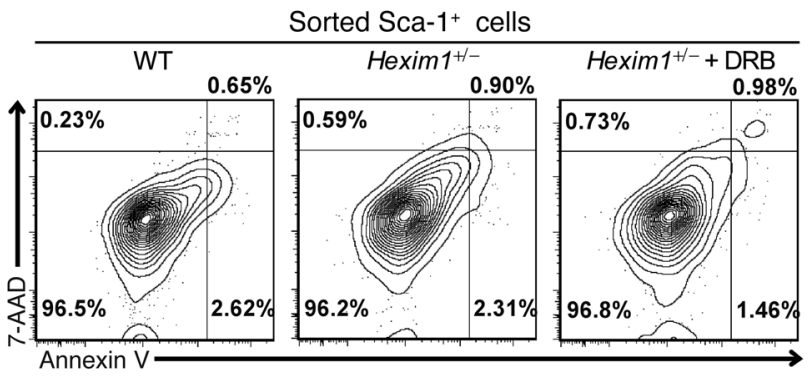

F

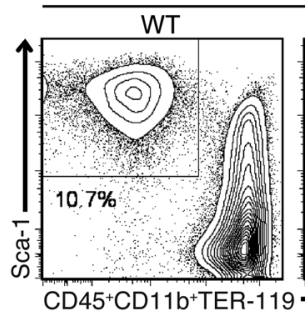

CD31-Integrin- $\alpha_{7}^{-}$gate
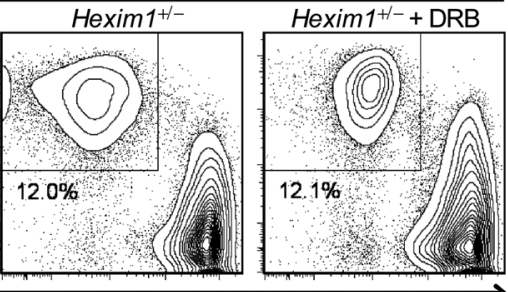

\section{Figure 3}

Hexim $1^{+/-}$satellite cells exhibit enhanced proliferation during regeneration. (A and $\left.\mathbf{B}\right)$ Representative plots and statistics showing cell cycle status of (A) sorted satellite cells and (B) Sca- $1^{+}$cells from injured and contralateral control muscles 4 days after injury. $G_{0}, G_{1}$, and $S / G_{2} / M_{\text {cells are }}$

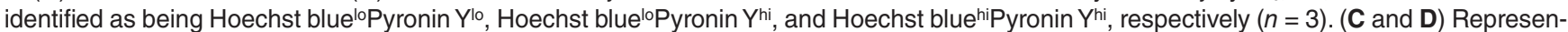
tative plots showing apoptosis of (C) sorted satellite cells and (D) Sca-1+ cells from injured and contralateral control muscles 4 days after injury. $(\mathbf{E}$ and $\mathbf{F})$ Percentages of $(\mathbf{E})$ satellite cells and $(\mathbf{F})$ Sca- $1^{+}$cells in injured and contralateral control muscles 4 days after injury. ${ }^{*} P<0.05$. Plots were representative of 3 independent experiments. Numbers represent percentages of cells in each gate or quadrant.

tosis was observed in Hexim $1^{+/-}$cultures in DM (Figure 6F), ruling out the possibility of rapid apoptosis of differentiated cells. These data show that induction of HEXIM1 expression inhibits satellite cell proliferation and triggers myogenic differentiation in vitro.
We further examined the effect of HEXIM1 induction on regeneration in vivo by intramuscular injection of HMBA into muscles at different regeneration stages and assessment of muscle weights and cross-section areas 50 days after injury (Supplemental Figure 7). 
A

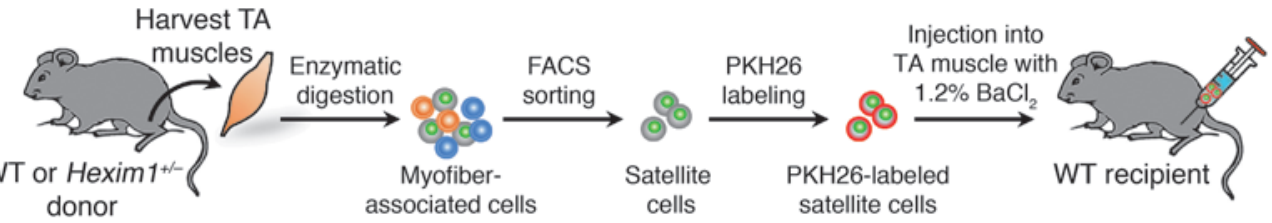

B

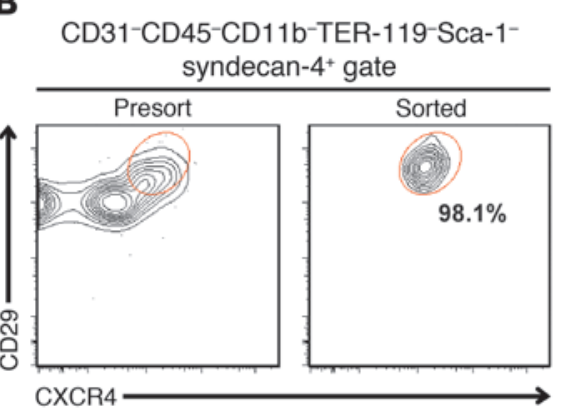

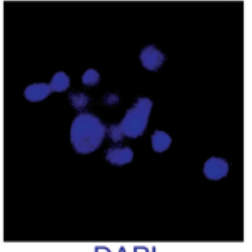

DAPI

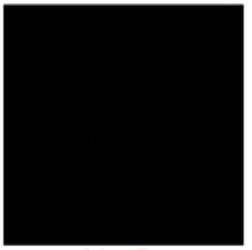

MyoD

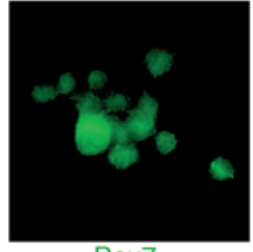

Pax7

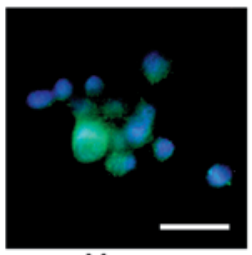

Merge

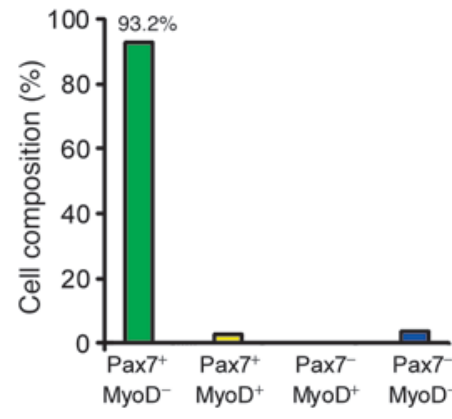

F CD31-CD45-CD11b-TER-119-Sca-1- gate

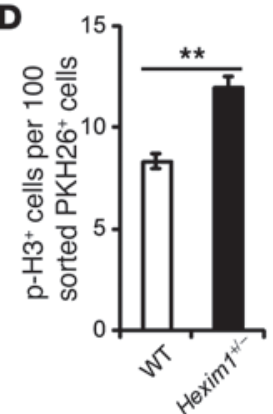

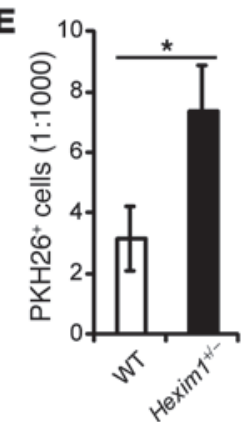

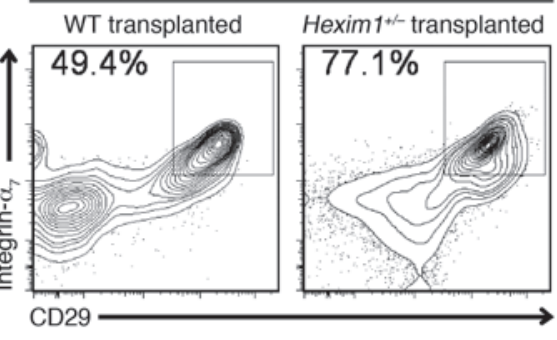

I

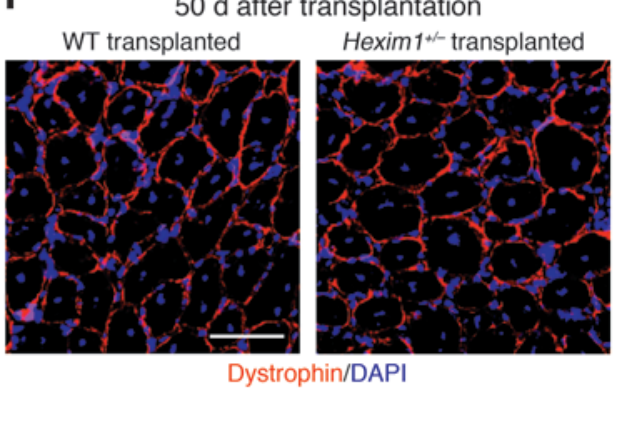

$50 \mathrm{~d}$ after transplantation

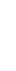

H

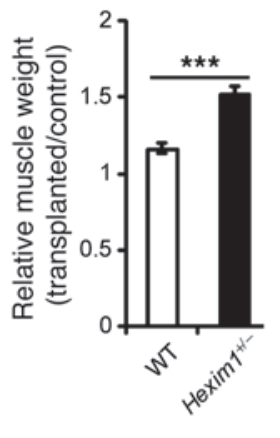

H
G

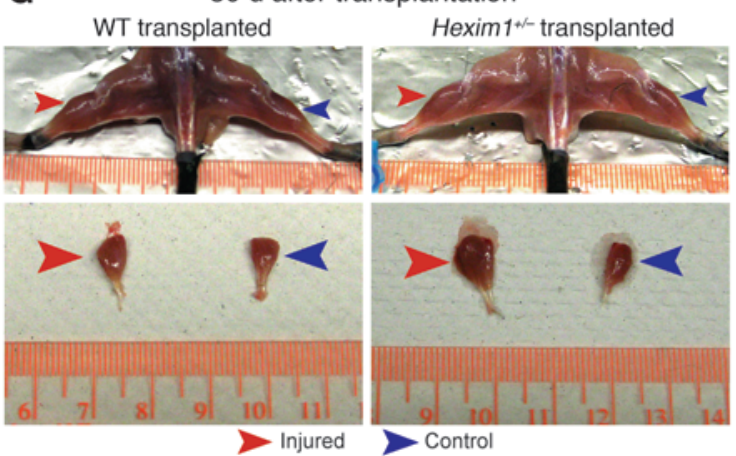

$50 \mathrm{~d}$ after transplantation Integrin- $\alpha_{7}{ }^{+} \mathrm{CD} 29+\mathrm{PKH} 26^{+}$gate

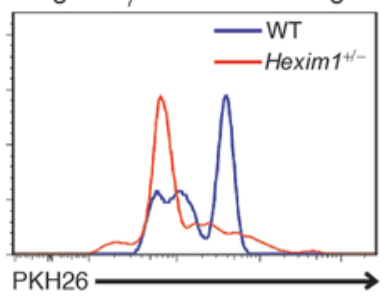

\section{Figure 4}

Transplanted Hexim $1^{+/}$satellite cells undergo enhanced expansion and improve muscle regeneration. (A) Scheme of satellite cell transplantation. (B) Purity and composition of freshly sorted cells. 466 out of 500 analyzed cells from 3 sorting experiments were Pax $7^{+} \mathrm{MyoD}^{-}$. Scale bar: $20 \mu \mathrm{m}$. (C) PKH26 signal of transplanted cells 3 days after transplantation. Host cells were excluded from analysis by PKH26 gating shown in Supplemental Figure 5D. (D) Percentages of phospho-histone $\mathrm{H}^{+}\left(\mathrm{p}-\mathrm{H} 3^{+}\right)$cells among PKH26 $6^{+}$cells 3 days after transplantation $(n=6)$. (E) PKH26 $6^{+}$ cells per 1,000 viable cells 3 days after transplantation $(n=6)$. (F) Percentages of satellite cells in recipient muscles 3 days after transplantation. (G) Transplanted and contralateral TA muscles 50 days after transplantation. (H) Weights of transplanted TA muscles normalized to those of contralateral muscles 50 days after injury $(n=6)$. (I) Muscle sections 50 days after transplantation stained for dystrophin (red). Scale bar: $80 \mu \mathrm{m}$. ${ }^{\star} P<0.05 ;{ }^{* \star} P<0.01 ;{ }^{* \star} P<0.001$. Histograms and plots were representative of 6 independent transplantation experiments. 
A

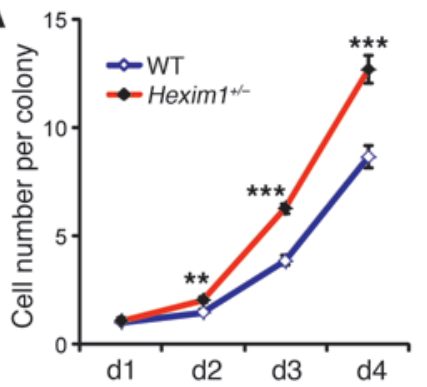

C

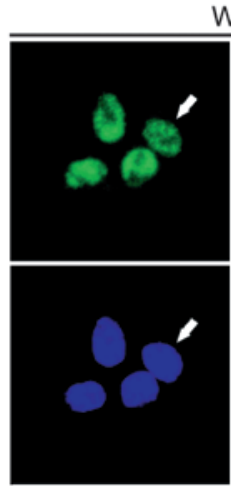

WT

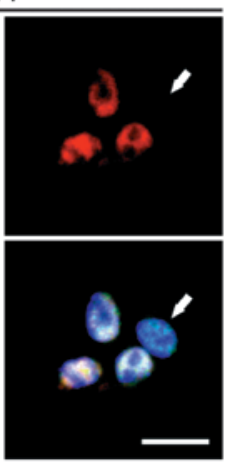

Pax7/MyoD/DAPI

E

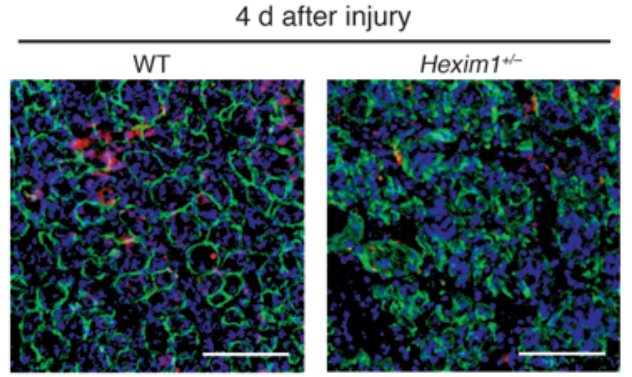

Laminin/myogenin/DAPI

B
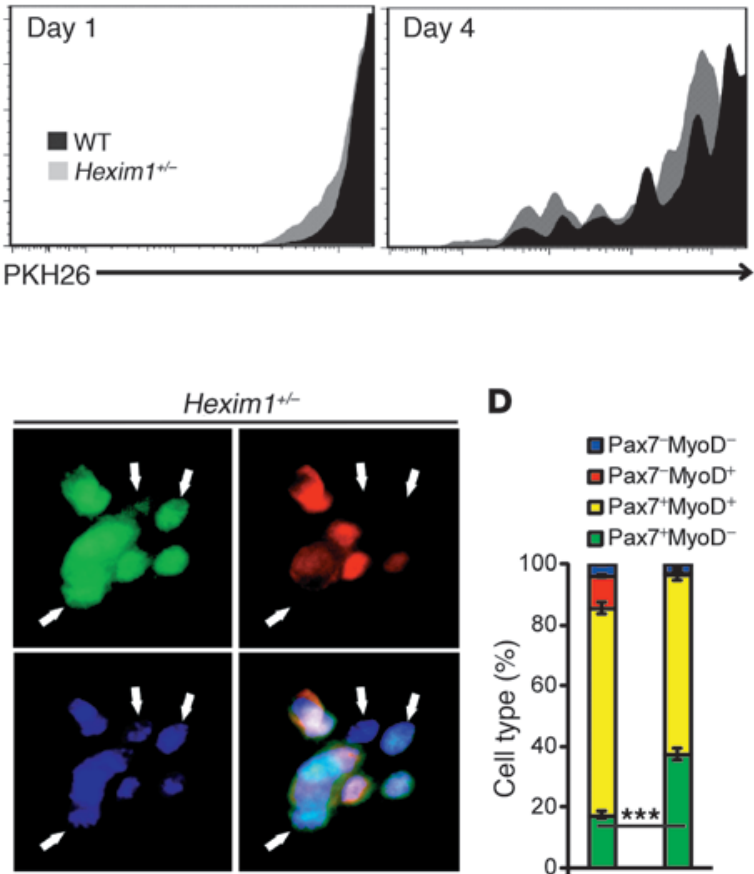

D

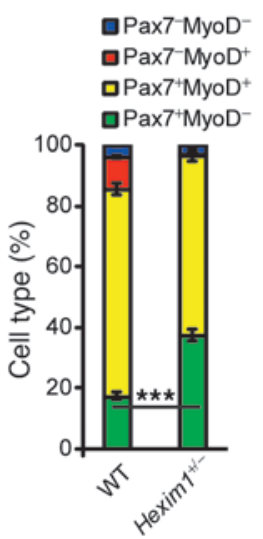

$\mathbf{F}$

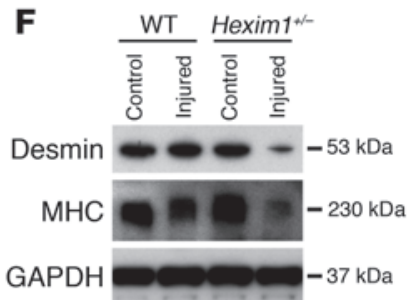

Figure 5

Hexim $1^{+/}$satellite cells exhibit enhanced proliferation over differentiation. (A) Average numbers of cells per colony from 20 single cellderived colonies. Data points represent days $1,2,3$, and 4 of culture in GM $(n=6)$. (B) Histogram showing PKH26 signals of sorted cells cultured in GM for 1 or 4 days. (C) Single cell-derived colonies stained for Pax7 (green) and MyoD (red). Arrows indicate $\mathrm{Pax} 7^{+} \mathrm{MyoD}-$ cells. Scale bar: $20 \mu \mathrm{m}$. (D) Percentages of cells in satellite cells cultured for 3 days in GM. (E) Muscle sections 4 days after injury stained for laminin (green) and myogenin (red). Laminin outlines regenerating myofibers, and myogenin marks differentiating myogenic cells. Scale bar: $100 \mu \mathrm{m}$. (F) Immunoblots of desmin and $\mathrm{MHC}$ in injured and contralateral control muscles 4 days after injury. (G) Myh3 mRNA levels 4 days after injury $(n=3)$. (H) Percentages of transplanted cells retaining satellite cell markers 3 days after transplantation. (I) Percentages of myogenin+ cells among PKH26+ cells 3 days after transplantation $(n=3)$. ${ }^{\star} P<0.05 ;{ }^{* \star} P<0.01 ;{ }^{* \star *} P<0.001$. Plots were representative of 6 independent experiments.

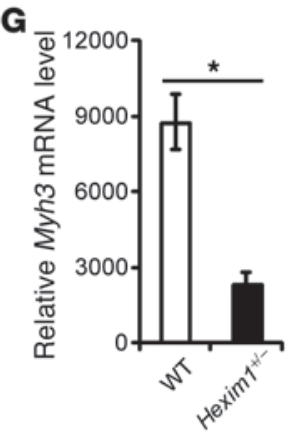

H CD31-CD45-CD11b-TER-119-Sca-1-PKH26+ gate
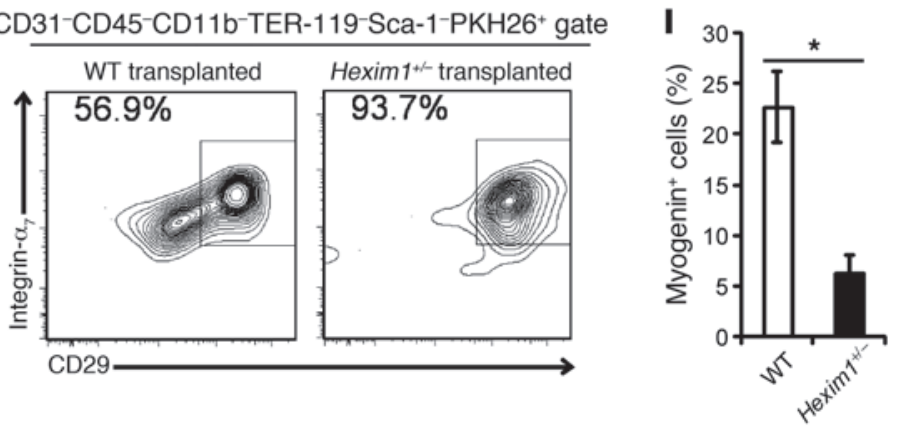

Administration of HMBA alone into uninjured muscles did not affect injured-to-control ratios of wet weights or cross-section areas (Figure 7, A and B), ruling out any effect due to HMBA administration independent of muscle injury. However, administration of HMBA at the time of injury reduced injured-to-control ratios of wet weights and cross-section areas in injured Hexim $1^{+/-}$ muscles by $30 \%$ and $27 \%$, respectively (Figure $7, \mathrm{~A}$ and B). There were more differentiating myoblasts and nascent myofibers after HMBA treatment (Figure 7, C and D). Similarly, following HMBA treatment, injured WT muscles also had $31 \%$ and $28 \%$ reductions in injured-to-control ratios of wet weights and cross-section areas, respectively (Figure 7, A and B). However, HMBA treatment at the later stages of regeneration produced less effect on the outcome of regeneration (Supplemental Figure 7 and Figure 7, A and B). These results support the conclusion that HEXIM1 regulates the expansion of satellite cells only during the early stages of regeneration.

HEXIM1 regulates muscle regeneration by limiting $P$-TEFb activity. To establish the relationship between P-TEFb and satellite cell proliferation, we first examined the activity of $\mathrm{P}-\mathrm{TEFb}$ in satellite cell cultures by measuring the phosphorylation level of Ser2 
A

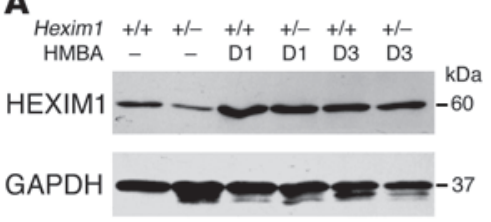

B

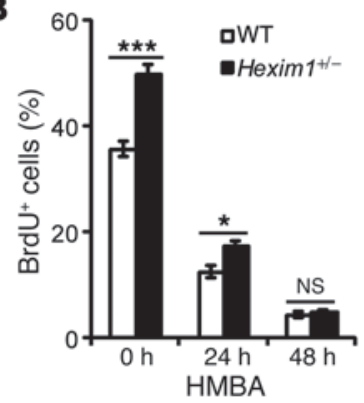

C

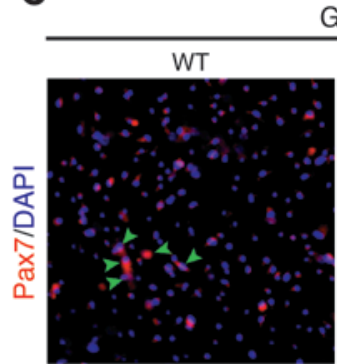

GM
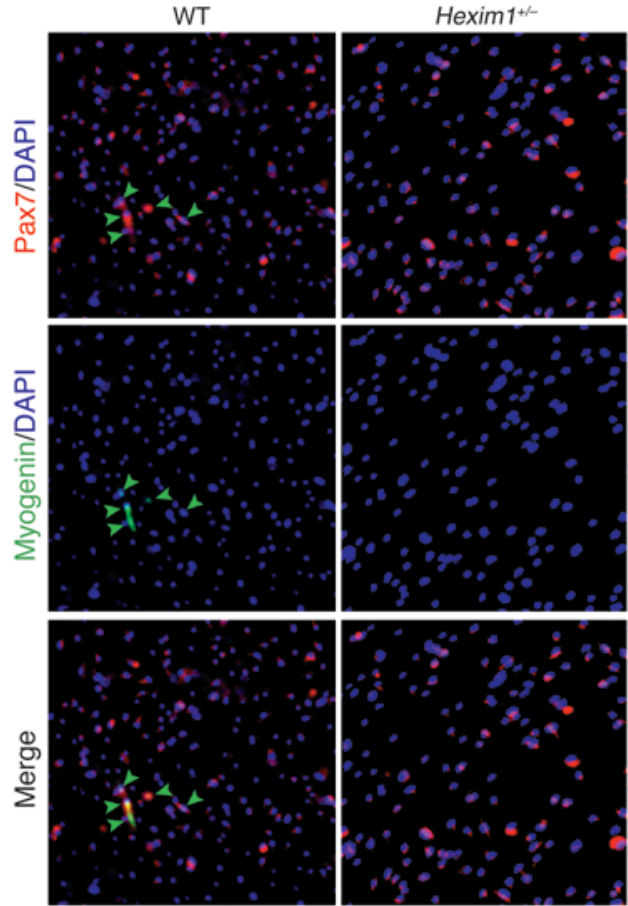

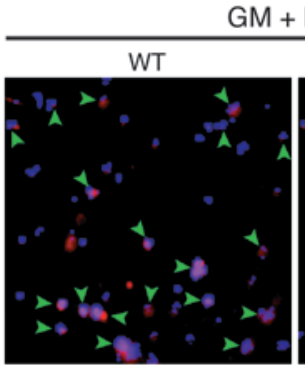

$\mathrm{GM}+\mathrm{HMBA}$
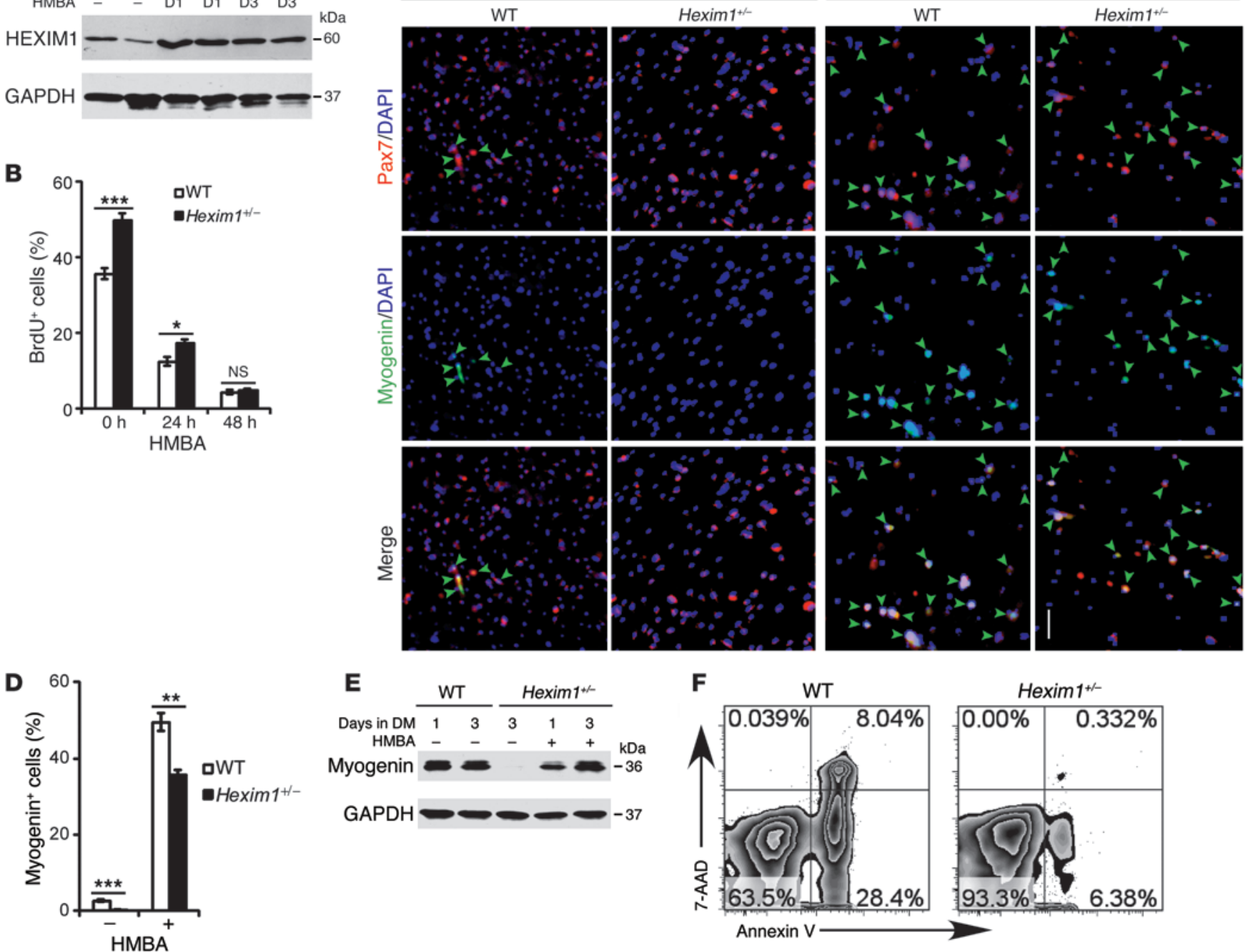

Figure 6

HMBA-induced HEXIM1 expression restrains satellite cell proliferation and promotes differentiation. (A) Immunoblots of satellite cells cultured in GM for the indicated period of time with or without $10 \mathrm{mM} \mathrm{HMBA}$. (B) BrdU incorporation of satellite cells cultured in GM with HMBA for the indicated time periods. 200 nuclei per slide were counted ( $n=6$ slides). (C) Satellite cells cultured in GM for 3 days stained for myogenin (green) and Pax7 (red) and (D) percentages of differentiating cells (myogenin+) $(n=6)$. (C and D) Green arrowheads indicate differentiating cells. Scale bar: $40 \mu \mathrm{m}$. (E) Immunoblots of satellite cells cultured in DM. (F) Apoptosis of satellite cells cultured in DM for 1 day. ${ }^{*} P<0.05 ;{ }^{*} P<0.01$; ${ }^{* * *} P<0.001$. Plots were representative of 3 independent experiments.

and CDK9 activity (45). The majority of phosphorylated Ser2 ( $\mathrm{p}$-Ser2) signals were found in proliferating $\left(\mathrm{Ki}^{\circ} 7^{+}\right)$cells (Figure $8 \mathrm{~A})$. Hexim $^{+/-}$cultures showed $50 \%$ higher $\mathrm{p}$-Ser2 signals and CDK9 activities (Figure 8B) and 49\% higher proliferation rates (Figure 8C) than WT cultures. Treatment with 5,6-dichlorobenzimidazone-1- $\beta$-D-ribofuranoside (DRB), a chemical inhibitor of P-TEFb independent of HEXIM1 (46), reduced proliferation rates of cultures of both genotypes to a comparable level (Figure 8C). Intramuscular administration of DRB to injured muscles inhibited CDK9 kinase activity and reduced proliferation rates of Hexim $^{+/-}$satellite cells to WT levels (Supplemental Figure 8A and Supplemental Figure $3 \mathrm{~A})$. Sca- $1^{+}$cells, which proliferated much more slowly than satellite cells, were not affected by DRB during regeneration (Figure $3 \mathrm{~B}$ ). Similar inhibition of $\mathrm{P}-\mathrm{TEFb}$ and prolif- eration was observed upon HMBA treatment (Figure 8, D-F). Furthermore, upon treatment with IL-6, an activator of satellite cell proliferation (47), HEXIM1 was released from P-TEFb complexes more rapidly in Hexim $1^{+/}$cultures than in WT controls (Figure 8G). 7SK RNA and mRNA of other P-TEFb components, Cdk9 and cyclin T1 (Ccnt1), remained unaltered in Hexim 1 ${ }^{+/-}$muscles (Supplemental Figure 8B). The expression of several 7SK target genes, which are regulated by P-TEFb-independent mechanisms (48), was also comparable in control and injured muscles between the 2 genotypes (Supplemental Figure 8B). These data demonstrate that haplodeficiency of Hexim 1 enhances proliferation of satellite cells by relieving the inhibition of P-TEFb.

In contrast to proliferating cells, mature myonuclei located in myotubes exhibited little P-TEFb activity (Figure 9A). Over the 
A
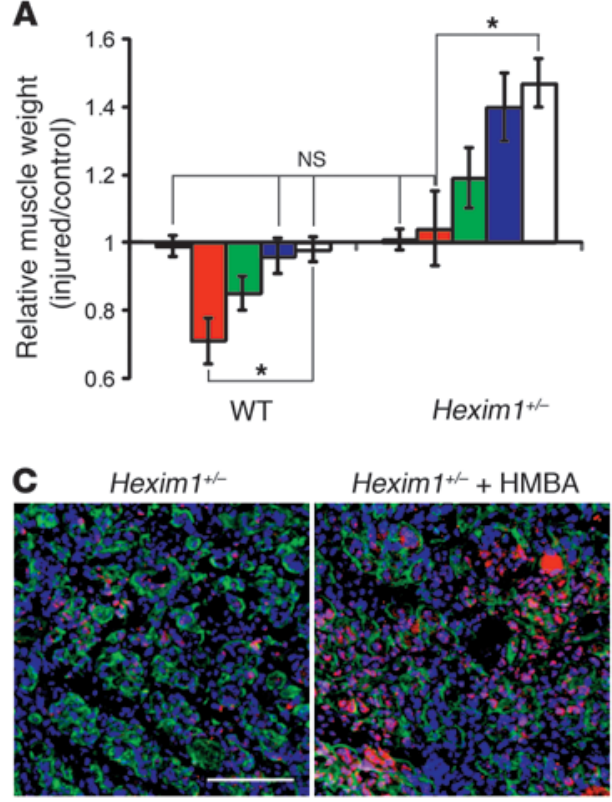

Laminin/myogenin/DAPI
B

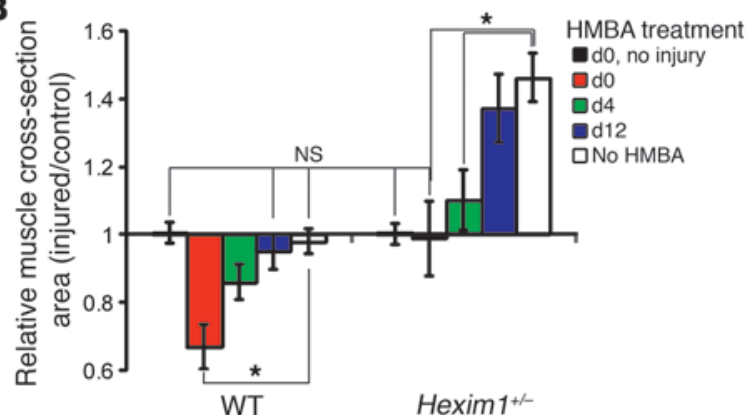

Hexim1 $^{+-}$

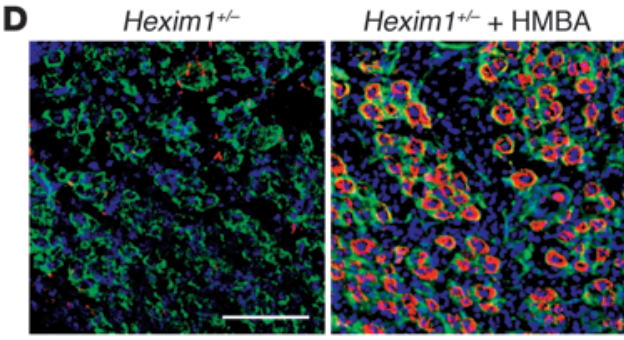

Laminin/MHC/DAPI

Figure 7

HEXIM1 overexpression reduces regenerative capacity by promoting early differentiation of satellite cells. (A and B) Wet weights and cross-section areas of injured TA muscles normalized to those of contralateral controls 50 days after injury. HMBA was administered at indicated time points, as illustrated in Supplemental Figure $7(n=3)$. (C and D) Sections of Hexim1+/-TA muscles 4 days after injury stained for $(\mathbf{C})$ laminin $($ green) and myogenin (red) or (D) laminin (green) and MHC (red). Scale bar: $100 \mu \mathrm{m} .{ }^{*} P<0.05$.

period of cell cycle exit and differentiation, p-Ser2 levels gradually decreased along with those of cell cycle protein cyclin D1 (Figure 9B). Unlike WT controls, Hexim $1^{+/}$cells downregulated p-Ser2 levels only after 3 days in GM (Figure 9C), due to the acquisition of quiescence. Less HEXIM1 was recruited to P-TEFb complex in Hexim $1^{+/-}$cultures upon DM treatment (Figure 9D). These data indicate a pro-differentiation function of HEXIM1 via inhibition of $\mathrm{P}-\mathrm{TEFb}$ activity.

To validate the above results in vivo, we analyzed p-Ser2 level and CDK9 activity in injured muscles. Low p-Ser2 levels and few $\mathrm{p}$-Ser2 ${ }^{+}$satellite cells were observed in muscles before injury or 50 days after injury, while significant elevation of CDK9 activity and $\mathrm{p}$-Ser 2 level and more $\mathrm{p}$-Ser $2^{+}$satellite cells were observed in muscles 4 and 12 days after injury (Supplemental Figure 8, C-F), revealing a transient increase in P-TEFb activity. Higher CDK9 activity and p-Ser 2 level and 3 -fold more p-Ser $2^{+}$satellite cells were found in injured Hexim $1^{+/}$muscles than in injured WT muscles 4 and 12 days after injury (Supplemental Figure 8, C to F). In agreement with the higher CDK9 activity and p-Ser2 level during regeneration, the amount of HEXIM1 associated with P-TEFb decreased after injury in both WT and Hexim $1^{+/-}$muscles, indicating that muscle injury triggered a release of HEXIM1 from P-TEFb (Supplemental Figure 8C). Less HEXIM1 was associated with $\mathrm{P}-\mathrm{TEFb}$ in Hexim ${ }^{+/-}$muscles than in WT muscles at corresponding injury stages, as a result of the lower cellular availability of HEXIM1 in Hexim 1 ${ }^{+/}$cells (Supplemental Figure 8C). These data corroborate the above findings of enhanced P-TEFb activity as a result of insufficient HEXIM1 level.

In summary, haplodeficiency of Hexim1 in satellite cells causes robust and prolonged activation of $\mathrm{P}-\mathrm{TEFb}$ after injury as a result of relieved HEXIM1-dependent inhibition of P-TEFb, which in turn promotes proliferation and expansion of the satellite cell pool and results in enhanced skeletal muscle regeneration (Figure 10).

\section{Discussion}

HEXIM1 and its partner P-TEFb have been demonstrated to regulate a wide range of physiological and pathological processes, such as embryonic and cardiac development, cardiomyocyte hypertrophic growth, and human immunodeficiency virus 1 infection (28). Such diverse functions of the HEXIM1/P-TEFb pathway are believed to be a result of its role in the regulation of global gene expression during transcription pausing escape and productive elongation $(28,29)$. Recently, several reports showed evidence of gene-specific recruitment of P-TEFb by various transcription factors, indicating that $\mathrm{P}-\mathrm{TEFb}$ not only permits global transcription elongation but also could promote the expression of specific genes by shortening or bypassing transcription pausing $(49,50)$. MyoD, the master transcription factor of myogenic differentiation, has been shown to interact with P-TEFb during the transcription of MyoD target genes, and P-TEFb activity is required for the MyoDmediated differentiation of satellite cells in vitro $(33,34)$. In this study, we further demonstrate that skeletal muscle regeneration, which is achieved by the de novo formation of myofibers from the satellite cell pool, is controlled by the HEXIM1/P-TEFb pathway that regulates satellite cell expansion after injury. Mechanistically, during regeneration satellite cells undergo proliferation and then terminal differentiation, both of which are implicated to be modulated by P-TEFb by in vitro studies based on nonmyogenic cell lines (44). Here, we show that relieved inhibition of P-TEFb by HEXIM1 promotes proliferation and expansion while repressing early myogenic differentiation of satellite cells during skeletal muscle regeneration, which substantiates the role of HEXIM1/P-TEFb as a gen- 
A

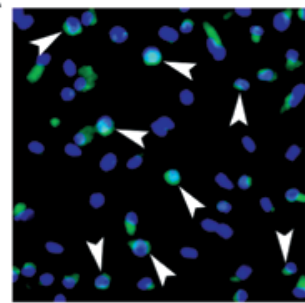

p-Ser2/DAPI

D
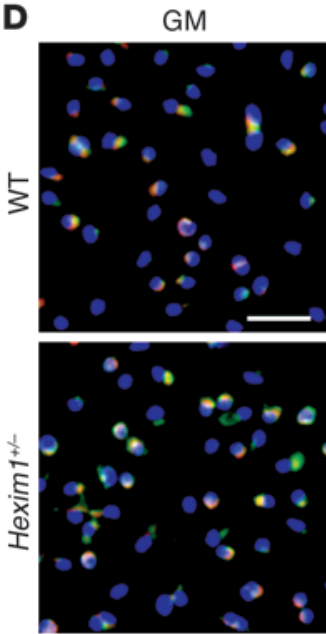

p-Ser2/Ki67/DAPI

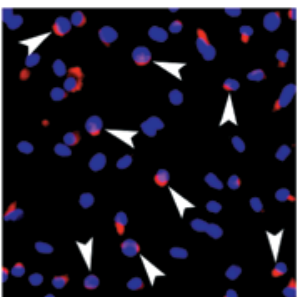

Ki67/DAPI
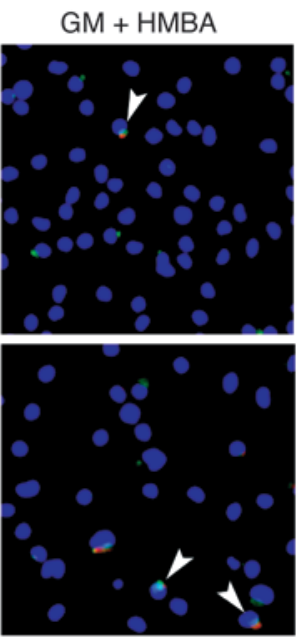

B

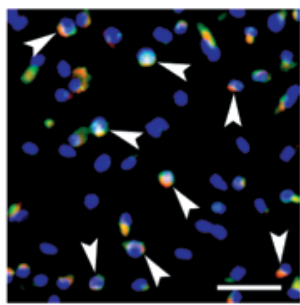

Merge

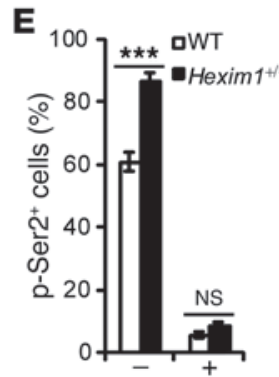

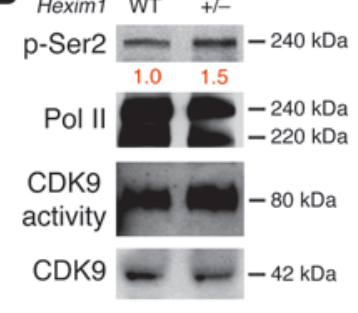

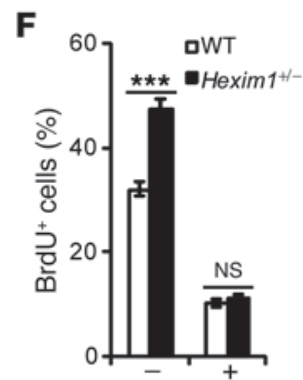

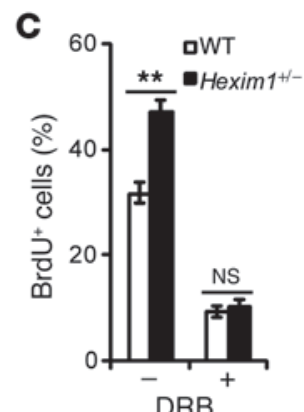

DRB

G

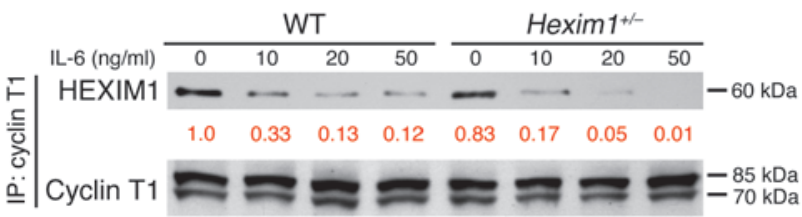

\section{Figure 8}

P-TEFb activation after HEXIM1 dissociation is required for satellite cell proliferation. (A) WT satellite cells cultured in GM for 3 days and stained for p-Ser2 (green) and Ki67 (red). Arrowheads indicate colocalization of Ki67 and p-Ser2 signals. Scale bar: $40 \mu \mathrm{m}$. (B) Immunoblots of satellite cells cultured in GM for 3 days. Red numbers indicate band intensity relative to that of WT. Pol II and CDK9 served as the control for p-Ser2 and CDK9 kinase activity, respectively. (C) BrdU incorporation in satellite cells cultured in GM with $50 \mu \mathrm{M}$ DRB for 24 hours $(n=6)$. (D-F) Satellite cells cultured in GM with HMBA for 3 days (D) stained for p-Ser2 (green) and Ki67 (red) and (E) quantitated for percentages of p-Ser2+ cells and (F) BrdU incorporation rates. Arrowheads point to cells in cell cycle after HMBA treatment $(n=6)$. Scale bar: $40 \mu \mathrm{m}$. (G) Immunoblots of satellite cells cultured in GM with indicated concentrations of IL-6 for 6 hours and immunoprecipitated with cyclin T1 antibody. Cyclin T1 served as control for immunoprecipitation. Red numbers below lanes indicate band intensity relative to that of untreated WT cells. ${ }^{* \star} P<0.01 ;{ }^{* \star *} P<0.001$.

eral regulator of cell proliferation and differentiation (44). In light of this, we speculate that other regenerative processes involving a proliferation-to-differentiation switch analogous to that of satellite cells, such as adult neurogenesis and wound healing, may be regulated similarly by HEXIM1/P-TEFb. Considering this promising potential, current knowledge regarding the precise effect of HEXIM1/P-TEFb on context-specific transcription is insufficient and calls for further investigation of downstream targets of HEXIM1/P-TEFb and their physiological significance.

Upregulation of $\mathrm{P}-\mathrm{TEFb}$ in Hexim $1^{+/-}$mice increases muscle mass and myofiber number without hypertrophic growth after injury, which is a potentially beneficial effect. In contrast, intramuscular injection of satellite cells after injury increases the size and mass of regenerated muscles and induces hypertrophic growth of myofibers (16). This difference could be explained by the fact that intramuscular injection of satellite cells increases satellite cell density only in a specific region near the delivery site due to limited migration capability of transplanted satellite cells (20), whereas the enrichment of satellite cells by enhancing their proliferation in our model was relatively uniform throughout the muscle. More- over, due to the large amount of cells delivered during injection, these satellite cells may not be completely consumed by the early regeneration process and thus could serve as an extra myogenic source at the later stages of regeneration. Therefore, enhanced proliferation in $\mathrm{Hexim}^{+/-}$muscles is unlikely to result in muscle hypertrophy as induced by injection of satellite cells.

Blockade of myostatin, a powerful inhibitor of skeletal muscle growth, enhances global myogenesis, including both muscle maintenance and regeneration (51), while haplodeficiency of Hexim 1 specifically promotes muscle regeneration after injury. The preserved functional capacity of Hexim $1^{+/}$muscles also indicates a clear mechanistic departure from that of myostatin blockade where there is reduced functional capacity (52). It has been shown that muscle function is not only proportional to the muscle size but also depends on the myonuclei density in each myofiber, which is maintained by satellite cell differentiation and fusion with myofibers (53). Others have reported that satellite cells undergo limited self-renewal by asymmetric division during muscle growth and maintenance but proliferate symmetrically with vigorous self-renewal and expansion during regeneration $(39,54)$. In fact, 
A
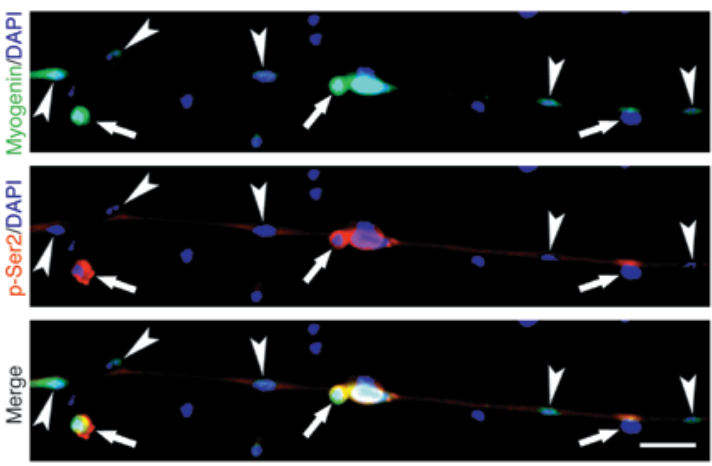

B

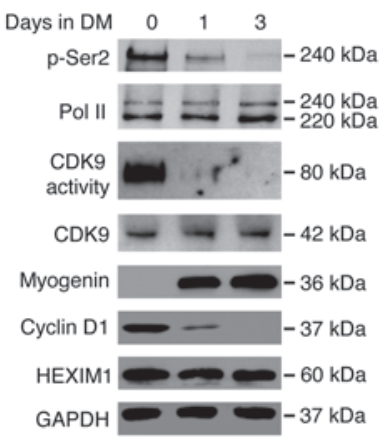

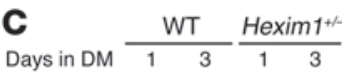
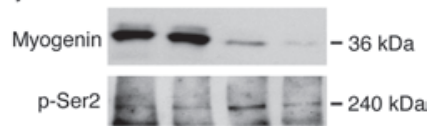

GAPDH $-37 \mathrm{kDa}$

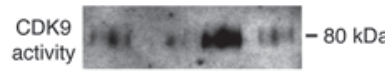

CDK9 $--1-42 \mathrm{kDa}$

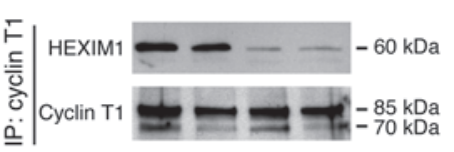

the cytokines secreted by infiltrating immune cells during early regeneration, which can activate $\mathrm{P}-\mathrm{TEFb}$ to promote the rapid expansion of the satellite cell pool, are present at much lower levels under resting conditions (1), rendering the reduced HEXIM1 level sufficient to maintain normal P-TEFb activity for the maintenance of the satellite cell pool. Therefore, we reasoned that haplodeficiency of Hexim1 does not affect muscle function during maintenance, due to low stimulation of the HEXIM1/P-TEFb pathway under resting conditions, but promotes muscle regeneration and functional recovery by augmenting the activation of P-TEFb, leading to rapid expansion and accumulation of satellite cells after injury. In contrast, recent reports showed that myostatin inhibition-induced hypertrophy was independent of satellite cell activity and possibly resulted from altered metabolism of muscle proteins (55), and this may explain the additional effect of myostatin inhibition on muscle maintenance.

Transplantation of satellite cells is a promising therapy to cure muscular dystrophies and promote muscle regeneration, while several obstacles, including the large numbers of satellite cells required to regenerate sufficient myofibers and the rapid loss of proliferative and regenerative potential after ex vivo expansion, prevent its wide application $(10,20,21)$. We have shown that cultured satellite cells proliferate at an optimal rate, without losing stem cell characteristics or regenerative potential when the cellular HEXIM1 protein level is reduced by half. Spontaneous cell cycle exit and early myogenic differentiation, a common but undesirable phenomenon in therapeutic satellite cell cultures, were rarely observed in Hexim $1^{+/}$ cultures. Furthermore, transplantation of Hexim $1^{+/-}$satellite cells leads to enhanced expansion of the satellite cell pool after injury and improves the outcome of muscle regeneration more effectively than transplantation of WT satellite cells. Therefore, lowering

\section{Figure 9}

Insufficient inhibition of P-TEFb by HEXIM1 enhances satellite cell proliferation by suppressing myogenic differentiation. (A) WT satellite cells cultured in DM for 3 days stained for myogenin (green) and p-Ser2 (red). Red background outlines the myotube. Arrows indicate myonuclei undergoing fusion into the myotube. Arrowheads point to differentiated myonuclei located inside the myotube. Scale bar: $40 \mu \mathrm{m}$. (B) Immunoblots of WT satellite cells cultured in DM for 0,1 , or 3 days. Pol II and CDK9 served as the control for p-Ser2 and CDK9 kinase activity, respectively. Immunoblots of (C) whole lysate and (D) lysates immunoprecipitated with cyclin T1 antibody from satellite cells cultured in DM for 1 or 3 days. Cyclin T1 served as the control for immunoprecipitation.

the cellular HEXIM1 protein level could be a useful approach to improve the efficacy of therapeutic satellite cell cultures.

It is noteworthy that cardiomyocyte-specific hyperactivation of P-TEFb by overexpression of cyclin T1 leads to hypertrophic growth of cardiomyocytes and accelerates the progression of heart failure $(45,56)$. However, because of the relatively low numbers of successfully engrafted satellite cells, as compared with the endogenous satellite cell pool as shown in clinical trials (10), it is possible that only a small fraction of myonuclei in the regenerated myofibers could be derived from transplanted satellite cells that have reduced HEXIM1 levels. It is also conceivable that WT myonuclei could increase Hexim 1 expression to compensate for the Hexim $1^{+/-}$ myonuclei located in the same myofiber, which therefore maintains a normal level of overall gene expression and protein synthesis. In fact, the injured WT muscles receiving Hexim $1^{+/-}$satellite cell transplantation did not develop hypertrophy during regeneration, despite the significant increase in size and myofiber numbers after regeneration. In summary, our findings suggest that transient inhibition of HEXIM1 may improve the ex vivo expansion of satellite cells as well as the therapeutic efficacy of satellite cell transplantation following ex vivo cultures and that controlled inhibition of HEXIM1 in vivo, if it has no deleterious side effect, could possibly benefit patients with muscular disorders.

\section{Methods}

Mice. Mice carrying a targeted deletion of the Hexim1 gene were generated, as described previously (57), and maintained on 129S/SvEv background. Fourto twelve-week-old age- and sex-matched mice were used for experiments.

Muscle injury. Skeletal muscle injury was induced by injection of $50 \mu \mathrm{l}$ $1.2 \% \mathrm{BaCl}_{2}$ (Sigma-Aldrich) dissolved in saline into the left TA muscle, as described previously (37). The extent of injury was determined by immunofluorescence of dystrophin and laminin staining. Only muscles with more than $90 \%$ myofibers injured were further analyzed. HMBA treatment was performed with injection of $50 \mu \mathrm{l}$ of $200 \mathrm{mM} \mathrm{HMBA}$ (Sigma-Aldrich) in saline or $1.2 \% \mathrm{BaCl}_{2}$ solution into the target muscle at the indicated time point (see Supplemental Figure 7), followed by a single i.p. injection of $50 \mu \mathrm{l}$ of $200 \mathrm{mM}$ HMBA 2 days later. DRB treatment was performed with the injection of $50 \mu \mathrm{l}$ of $250 \mathrm{mM}$ DRB (Sigma-Aldrich) in $1.2 \% \mathrm{BaCl}_{2}$ solution into the target muscle.

Muscle functional measurement. Mice were deeply anesthetized via i.p. injection of ketamine-xylazine mixture (80 and $10 \mathrm{mg} / \mathrm{kg}$ ) (Sigma-Aldrich) and carefully monitored throughout the experiment. Additional doses were administered as needed to ensure no reflex response to toe pinch. The distal tendon of the TA muscle was dissected free from surrounding tissue, individually tied with 4.0 braided surgical silk, and then cut at the most distal end. The sciatic nerve was exposed, and all its branches were 


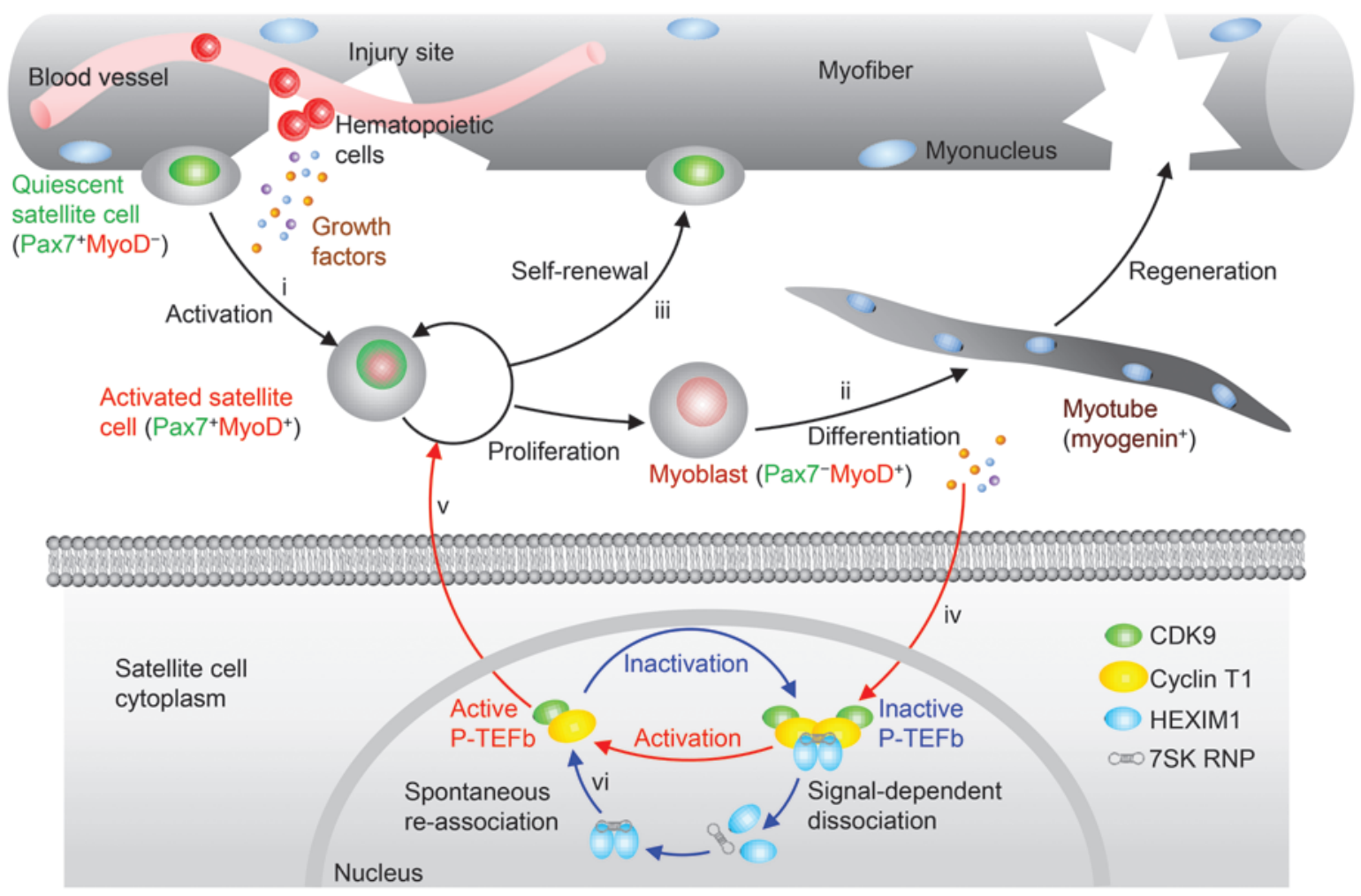

\section{Figure 10}

Model of HEXIM1/P-TEFb-dependent regulation of skeletal muscle regeneration. (i) Satellite cells are activated to proliferate by factors secreted in response to muscle injury, (ii) followed by myogenic differentiation and formation of new myofibers or (iii) return to quiescence to renew the satellite cell niche. P-TEFb, (iv) which is activated during regeneration, (v) promotes proliferation of satellite cells. (vi) HEXIM1 inhibits P-TEFb function by associating with the active P-TEFb complex and hence limits satellite cell proliferation and skeletal muscle regeneration.

cut except for the common peroneal nerve. The foot was secured to a platform, and the knee was immobilized using a stainless steel pin. The TA tendon was attached to the lever arm of a 305B dual-mode servomotor transducer (Aurora Scientific). TA muscle contractions were then elicited by stimulating the distal part of the sciatic nerve via bipolar electrodes, using supramaximal square-wave pulses of $0.02 \mathrm{~ms}$ (701A stimulator; Aurora Scientific). Data acquisition and control of the servomotors were conducted using a LabVIEW-based DMC program (version 5.202; Aurora Scientific). The muscle length was measured using digital calipers based on well-defined anatomical landmarks. The optimal muscle length $\left(\mathrm{L}_{\mathrm{o}}\right)$ was determined by incrementally stretching the muscle using micromanipulators until the maximum isometric twitch force was achieved. Three maximum isometric tetanic forces $\left(\mathrm{P}_{\mathrm{o}}\right)$ were determined using a train of $150-\mathrm{Hz}, 500-\mathrm{ms}$ supramaximal electrical pulses at the $\mathrm{L}_{\mathrm{o}}$ in the muscle, and the highest $\mathrm{P}_{\mathrm{o}}$ was recorded. A 2-minute resting period was allowed between each tetanic contraction. The normalized tetanic force $(\mathrm{N} / \mathrm{g})$ was calculated by dividing $\mathrm{P}_{\mathrm{o}}$ by the muscle weight.

Satellite cell and single myofiber isolation. Satellite cells were isolated from intact TA muscles following published methods (9) detailed in Supplemental Methods. Single myofibers were isolated and cultured as described previously (43).

Flow cytometry and cell sorting. Cells were incubated with an Fc blocking antibody (clone 2.4G2, BD Pharmingen) in staining buffer consisting of PBS (Gibco) and 3\% FBS (HyClone) for 20 minutes at $4^{\circ} \mathrm{C}$ and then stained with primary monoclonal antibodies for 30 minutes at $4^{\circ} \mathrm{C}$. Cells were washed with staining buffer followed by incubation with secondary antibodies, if necessary, for 20 minutes at $4^{\circ} \mathrm{C}$. 7-AAD (BD Pharmingen) was used to exclude nonviable cells from analysis. Antibodies and dilutions are provided in Supplemental Table 1. Apoptosis was determined using an Annexin V-eFluor 450 apoptosis detection kit (eBioscience). Cell cycle status was analyzed using Hoechst 33342 (Sigma-Aldrich) and Pyronin Y (Sigma-Aldrich) staining, as detailed in Supplemental Methods. Side population cells were identified by Hoechst 33342 exclusion and verified by adding $0.5 \mu \mathrm{g} / \mathrm{ml}$ verapamil (Sigma-Aldrich) into the staining buffer. Events were acquired on an LSR II or LSRFortessa flow cytometer (BD Biosciences) and analyzed using FlowJo (Tree Star). All gates and quadrants were drawn to give $\leq 1 \%$ positive cells in the sample stained with isotype control antibodies. $\mathrm{CD} 45^{+}$cells were separated by MACS using anti-CD45 microbeads (Miltenyi Biotec). Satellite cells and Sca- $1^{+}$cells were sorted on an Aria II cell sorter (BD Biosciences). Purity of the sorted cells was verified immediately after sorting.

Satellite cell labeling and transplantation. Freshly sorted satellite cells were labeled using a PKH26 red fluorescence cell linker kit (Sigma-Aldrich) following the manufacturer's instruction with modifications. PKH26 was diluted to a final concentration of $1 \times 10^{-6} \mathrm{M}(0.5 \times)$ for better viability, and $1 \%$ BSA (Fisher Bioreagents) was used as stop solution instead of serum to avoid activation of quiescent satellite cells. The labeling efficiency of WT and Hexim 1+/- cells was verified to be equal by flow cytometry immediately after labeling. Transplantation of labeled cells was performed by injection of 10,000 cells resuspended in $50 \mu \mathrm{l} 1.2 \% \mathrm{BaCl}_{2}$ into the left TA muscle of each WT mouse.

Satellite cell culture and assays. Satellite cells were plated on collagen Icoated plates or culture slides (BD BioCoat) and cultured in GM con- 
sisting of Ham's F-10 nutrient mixture (F10) (HyClone) with 20\% FBS, $10 \mathrm{mM}$ HEPES (Gibco), $5 \mathrm{ng} / \mathrm{ml} \mathrm{bFGF} \mathrm{(Invitrogen),} \mathrm{and} \mathrm{1 \%} \mathrm{penicillin/}$ streptomycin/amphotericin b (HyClone). Fresh bFGF was added to GM daily. Cells were passed onto new plates after 5 days or reaching $50 \%$ confluence. Fibroblasts in cultures of unsorted satellite cells were removed by preplating. Fibroblasts were distinguished under an inverted microscope during culturing and by Pax7 staining after fixation. Only cultures that contained less than $1 \%$ fibroblasts were used for experiment. Cultures not used after 4 passages were discarded. Procedures of assays are detailed in Supplemental Methods.

Histology and immunofluorescence. Histology and immunofluorescence analysis of muscle sections and cells were performed based on published methods $(9,38)$, as detailed in Supplemental Methods. Antibodies used in immunofluorescence are listed in Supplemental Table 1.

Quantitative real-time RT-PCR. Experimental procedures and conditions are detailed in Supplemental Methods. Each sample was run in triplicate, and raw results were normalized to the mean level of $18 \mathrm{~S}$ rRNA. The mean values of 3 raw results for the same gene from different animals were further calculated and expressed as fold induction relative to WT uninjured controls. Primers used in quantitative real-time RT-PCR are listed in Supplemental Table 2.

Immunoprecipitation and immunoblotting. Tissues and cells were lysed in Cellytic lysis reagent (Sigma-Aldrich) with protease inhibitors (Roche) and phosphatase inhibitors (Roche). Immunoprecipitation was performed using a reversible immunoprecipitation kit (Millipore) with appropriate isotype controls. Samples were resolved by SDS-PAGE and transferred to PVDF membranes (Invitrogen). Protein expression was visualized using horseradish peroxidase-conjugated secondary antibodies (Jackson ImmunoResearch Inc.) and enhanced chemiluminescence reagent (Amersham). Antibodies and dilutions are provided in Supplemental Table 1.

CDK9 kinase assay. CDK9 kinase assay was performed based on a published method (58), with a modified detection method based on immunoblotting. Briefly, CDK9 complex was immunoprecipitated with protein A-Sepharose beads using a spin column (Amersham). Reaction mixture consisting of $5 \mathrm{ng}$ recombinant GST-CTD (Sigma-Aldrich) and $10 \mu \mathrm{M}$ ATP
(Invitrogen) in $1 \times$ kinase buffer A (Invitrogen) was directly added to the beads and incubated at room temperature for 1 hour. GST-CTD and CDK9 were eluted by elution buffer (Amersham) and then subjected to immunoblotting. Antibodies and dilutions are provided in Supplemental Table 1.

ELISA. VEGF protein levels in injured muscles were measured by an ELISA Kit (R\&D Systems). Each sample was run in triplicate, and the mean of 3 raw results was calculated and expressed as pg of VEGF per $1 \mathrm{mg}$ total muscle protein.

Statistics. Values were expressed as mean \pm SEM. Statistical significance was assessed by a 2-tailed unpaired Student's $t$ test unless otherwise indicated. Differences were deemed statistically significant for $P$ values of less than 0.05 .

Study approval. All animal experiments were approved by the Institutional Animal Care and Use Committee of SUNY Downstate Medical Center.

\section{Acknowledgments}

We sincerely thank Katherine Hajjar and Gianna Ballon (Weill Cornell Medical College); Weifeng Xu, Meimei Shan, and Xiaodong Ge (Mount Sinai Medical Center); and Weijun Jin and Michael Wagner (SUNY Downstate Medical Center) for comments on experimental design and the article as well as Zhiqiang Li and Hui Jiang (SUNY Downstate Medical Center) for technical support. This work was supported in part by NIH grants HL073399 (to M.A.Q. Siddiqui) and AR052646 (to E.R. Barton), intramural SUNY funds (to M.A.Q. Siddiqui), and a research grant from the Wayne State University School of Medicine (to K. Chen).

Received for publication January 11, 2012, and accepted in revised form August 2, 2012.

Address correspondence to: M.A.Q. Siddiqui, 450 Clarkson Avenue, Box 5, Brooklyn, New York 11203, USA. Phone: 718.270.1014; Fax: 718.270.3732; E-mail: MAQ.Siddiqui@downstate.edu.

Bihui Huang's present address is: Department of Pharmacology, Yale University School of Medicine, New Haven, Connecticut, USA.
1. Charge SB, Rudnicki MA. Cellular and molecular regulation of muscle regeneration. Physiol Rev. 2004;84(1):209-238.

2. Tedesco FS, Dellavalle A, Diaz-Manera J, Messina G, Cossu G. Repairing skeletal muscle: regenerative potential of skeletal muscle stem cells. J Clin Invest. 2010;120(1):11-19.

3. McNally EM, Pytel P. Muscle diseases: the muscular dystrophies. Annu Rev Pathol. 2007;2:87-109.

4. Jarvinen TA, Jarvinen TL, Kaariainen M, Kalimo H, Jarvinen M. Muscle injuries: biology and treatment. Am J Sports Med. 2005;33(5):745-764.

5. Lu QL, et al. Functional amounts of dystrophin produced by skipping the mutated exon in the $\mathrm{mdx}$ dystrophic mouse. Nat Med. 2003;9(8):1009-1014.

6. Gregorevic P, et al. Systemic delivery of genes to striated muscles using adeno-associated viral vectors. Nat Med. 2004;10(8):828-834.

7. Cohn RD, et al. Angiotensin II type 1 receptor blockade attenuates TGF-beta-induced failure of muscle regeneration in multiple myopathic states. Nat Med. 2007;13(2):204-210.

8. Palacios D, et al. TNF/p38alpha/polycomb signaling to Pax7 locus in satellite cells links inflammation to the epigenetic control of muscle regeneration. Cell Stem Cell. 2010;7(4):455-469.

9. Conboy IM, Conboy MJ, Smythe GM, Rando TA. Notch-mediated restoration of regenerative potential to aged muscle. Science. 2003;302(5650):1575-1577.

10. Mendell JR, et al. Myoblast transfer in the treatment of Duchenne's muscular dystrophy. N EnglJ
Med. 1995;333(13):832-838.

11. Sampaolesi M, et al. Cell therapy of alpha-sarcoglycan null dystrophic mice through intra-arterial delivery of mesoangioblasts. Science. 2003; 301(5632):487-492.

12. Darabi R, et al. Functional skeletal muscle regeneration from differentiating embryonic stem cells. Nat Med. 2008;14(2):134-143.

13. Seale P, Sabourin LA, Girgis-Gabardo A, Mansouri A, Gruss P, Rudnicki MA. Pax7 is required for the specification of myogenic satellite cells. Cell. 2000;102(6):777-786.

14. Kuang S, Charge SB, Seale P, Huh M, Rudnicki MA. Distinct roles for Pax7 and Pax3 in adult regenerative myogenesis. J Cell Biol. 2006;172(1):103-113.

15. Montarras D, et al. Direct isolation of satellite cells for skeletal muscle regeneration. Science. 2005;309(5743):2064-2067.

16. Hall JK, Banks GB, Chamberlain JS, Olwin BB. Prevention of muscle aging by myofiber-associated satellite cell transplantation. Sci Transl Med. 2010; 2(57):57ra83.

17. Mokalled MH, Johnson AN, Creemers EE, Olson EN. MASTR directs MyoD-dependent satellite cell differentiation during skeletal muscle regeneration. Genes Dev. 2012;26(2):190-202.

18. Buckingham M, Relaix F. The role of Pax genes in the development of tissues and organs: Pax3 and Pax7 regulate muscle progenitor cell functions. Annu Rev Cell Dev Biol. 2007;23:645-673.

19. Rudnicki MA, Schnegelsberg PN, Stead RH, Braun
T, Arnold HH, Jaenisch R. MyoD or Myf-5 is required for the formation of skeletal muscle. Cell. 1993;75(7):1351-1359.

20. Kuang S, Rudnicki MA. The emerging biology of satellite cells and their therapeutic potential. Trends Mol Med. 2008;14(2):82-91.

21. Blau HM. Cell therapies for muscular dystrophy. N Engl J Med. 2008;359(13):1403-1405.

22. Doyle MJ, et al. Abcg2 labels multiple cell types in skeletal muscle and participates in muscle regeneration. J Cell Biol. 2011;195(1):147-163.

23. Vella JB, Thompson SD, Bucsek MJ, Song M, Huard J. Murine and human myogenic cells identified by elevated aldehyde dehydrogenase activity: implications for muscle regeneration and repair. PLoS One. 2011;6(12):e29226.

24. Dellavalle A, et al. Pericytes resident in postnatal skeletal muscle differentiate into muscle fibres and generate satellite cells. Nat Commun. 2011;2:499.

25. Seale P, Ishibashi J, Scime A, Rudnicki MA. Pax7 is necessary and sufficient for the myogenic specification of CD45+:Sca1+ stem cells from injured muscle. PLoS Biol. 2004;2(5):E130.

26. Huang F, Wagner M, Siddiqui MA. Structure, expression, and functional characterization of the mouse CLP-1 gene. Gene. 2002;292(1-2):245-259.

27. Yik JH, Chen R, Nishimura R, Jennings JL, Link AJ, Zhou Q. Inhibition of P-TEFb (CDK9/Cyclin T) kinase and RNA polymerase II transcription by the coordinated actions of HEXIM1 and 7SK snRNA. Mol Cell. 2003;12(4):971-982. 
28. Peterlin BM, Price DH. Controlling the elongation phase of transcription with P-TEFb. Mol Cell. 2006;23(3):297-305.

29. Yang Z, et al. Recruitment of P-TEFb for stimulation of transcriptional elongation by the bromodomain protein Brd4. Mol Cell. 2005;19(4):535-545.

30. Barboric M, Nissen RM, Kanazawa S, Jabrane-Ferrat $\mathrm{N}$, Peterlin BM. NF-kappaB binds P-TEFb to stimulate transcriptional elongation by RNA polymerase II. Mol Cell. 2001;8(2):327-337.

31. Rahl PB, et al. c-Myc regulates transcriptional pause release. Cell. 2010;141(3):432-445.

32. Nojima M, Huang Y, Tyagi M, Kao HY, Fujinaga $\mathrm{K}$. The positive transcription elongation factor $\mathrm{b}$ is an essential cofactor for the activation of transcription by myocyte enhancer factor 2. J Mol Biol. 2008; 382(2):275-287.

33. Simone C, et al. Activation of MyoD-dependent transcription by cdk9/cyclin T2. Oncogene. 2002; 21(26):4137-4148

34. Giacinti C, et al. Cdk9-55: a new player in muscle regeneration. J Cell Physiol. 2008;216(3):576-582.

35. Freter R, Osawa M, Nishikawa SI. Adult stem cells exhibit global suppression of RNA polymerase II serine-2 phosphorylation. Stem Cells. 2010; 28(9):1571-1580.

36. Galatioto J, Mascareno E, Siddiqui MA. CLP-1 associates with MyoD and HDAC to restore skeletal muscle cell regeneration. J Cell Sci. 2010; 123(Pt 21):3789-3795

37. Caldwell CJ, Mattey DL, Weller RO. Role of the basement membrane in the regeneration of skeletal muscle. Neuropathol Appl Neurobiol. 1990;16(3):225-238.

38. Shea KL, et al. Sprouty1 regulates reversible quiescence of a self-renewing adult muscle stem cell pool during regeneration. Cell Stem Cell. 2010; 6(2):117-129.

39. Kuang S, Kuroda K, Le Grand F, Rudnicki MA.
Asymmetric self-renewal and commitment of satellite stem cells in muscle. Cell. 2007;129(5):999-1010.

40. Sherwood RI, et al. Isolation of adult mouse myogenic progenitors: functional heterogeneity of cells within and engrafting skeletal muscle. Cell. 2004;119(4):543-554.

41. Sacco A, Doyonnas R, Kraft P, Vitorovic S, Blau HM Self-renewal and expansion of single transplanted muscle stem cells. Nature. 2008;456(7221):502-506.

42. Cornelison DD, Wilcox-Adelman SA, Goetinck PF, Rauvala H, Rapraeger AC, Olwin BB. Essential and separable roles for Syndecan-3 and Syndecan-4 in skeletal muscle development and regeneration. Genes Dev. 2004;18(18):2231-2236.

43. Zammit PS, Golding JP, Nagata Y, Hudon V, Partridge TA, Beauchamp JR. Muscle satellite cells adopt divergent fates: a mechanism for self-renewal? J Cell Biol. 2004;166(3):347-357.

44. He N, Pezda AC, Zhou Q. Modulation of a P-TEFb functional equilibrium for the global control of cell growth and differentiation. Mol Cell Biol. 2006;26(19):7068-7076.

45. Sano $M$, et al. Activation and function of cyclin T-Cdk9 (positive transcription elongation factor-b) in cardiac muscle-cell hypertrophy. Nat Med. 2002; 8(11):1310-1317.

46. Baumli S, Endicott JA, Johnson LN. Halogen bonds form the basis for selective P-TEFb inhibition by DRB. Chem Biol. 2010;17(9):931-936.

47. Serrano AL, Baeza-Raja B, Perdiguero E, Jardi M, Munoz-Canoves P. Interleukin- 6 is an essential regulator of satellite cell-mediated skeletal muscle hypertrophy. Cell Metab. 2008;7(1):33-44.

48. Eilebrecht S, Becavin C, Leger H, Benecke BJ, Benecke A. HMGA1-dependent and independent 7SK RNA gene regulatory activity. RNA Biol. 2011; 8(1):143-157.

49. Bai X, et al. TIF1gamma controls erythroid cell fate by regulating transcription elongation. Cell. 2010;142(1):133-143.

50. Yokoyama A, Lin M, Naresh A, Kitabayashi I, Cleary ML. A higher-order complex containing AF4 and ENL family proteins with P-TEFb facilitates oncogenic and physiologic MLL-dependent transcription. Cancer Cell. 2010;17(2):198-212.

51. Bogdanovich S, et al. Functional improvement of dystrophic muscle by myostatin blockade. Nature. 2002;420(6914):418-421.

52. Amthor $\mathrm{H}$, et al. Lack of myostatin results in excessive muscle growth but impaired force generation. Proc Natl Acad Sci U S A. 2007;104(6):1835-1840.

53. Qaisar R, Renaud G, Morine K, Barton ER, Sweeney HL, Larsson L. Is functional hypertrophy and specific force coupled with the addition of myonuclei at the single muscle fiber level? FASEB J. 2012; 26(3):1077-1085.

54. Shinin V, Gayraud-Morel B, Gomes D, Tajbakhsh $\mathrm{S}$. Asymmetric division and cosegregation of template DNA strands in adult muscle satellite cells. Nat Cell Biol. 2006;8(7):677-687.

55. Wang Q, McPherron AC. Myostatin inhibition induces muscle fibre hypertrophy prior to satellite cell activation. J Physiol. 2012;590(pt 9):2151-2165.

56. Sano $\mathrm{M}$, et al. Activation of cardiac Cdk9 represses PGC-1 and confers a predisposition to heart failure. EMBO J. 2004;23(17):3559-3569.

57. Huang F, Wagner M, Siddiqui MA. Ablation of the CLP-1 gene leads to down-regulation of the HAND1 gene and abnormality of the left ventricle of the heart and fetal death. Mech Dev. 2004; 121(6):559-572.

58. Yang X, Herrmann CH, Rice AP. The human immunodeficiency virus Tat proteins specifically associate with TAK in vivo and require the carboxyl-terminal domain of RNA polymerase II for function. J Virol. 1996;70(7):4576-4584. 